\title{
Representation and Weak Convergence of Stochastic Integrals with Fractional Integrator Processes*
}

\author{
James Davidson ${ }^{\dagger}$ \\ Nigar Hashimzade \\ University of Exeter \\ University of Reading
}

Final Revision, September 2008

Keywords: Stochastic integral, weak convergence, fractional Brownian motion. JEL Codes: C22, C32

\begin{abstract}
This paper considers the asymptotic distribution of the sample covariance of a nonstationary fractionally integrated process with the stationary increments of another such process - possibly, itself. Questions of interest include the relationship between the harmonic representation of these random variables, which we have analysed in a previous paper, and the construction derived from moving average representations in the time domain. Depending on the values of the long memory parameters and choice of normalization, the limiting integral is shown to be expressible as the sum of a constant and two Itô-type integrals with respect to distinct Brownian motions. In certain cases the latter terms are of small order relative to the former. The mean is shown to match that of the harmonic representation, where the latter is defined, and satisfies the required integration by parts rule. The advantages of our approach over the harmonic analysis include the facts that our formulae are valid for the full range of the long memory parameters, and extend to non-Gaussian processes.
\end{abstract}

${ }^{*}$ We thank, among others, Javier Hualde, Philipp Sibbertsen, Toni Stocker, Peter Phillips, Bent Nielsen, editor Robert Taylor, and three anonymous referees for discussion and comments on previous versions of the paper.

${ }^{\dagger}$ Corresponding author. Department of Economics, University of Exeter, Exeter EX4 4PU, UK. Email: james.davidson@exeter.ac.uk 


\section{Introduction}

Let $x_{t}$ and $y_{t}$ be linear processes having the $\mathrm{MA}(\infty)$ forms

$$
x_{t}=\sum_{j=0}^{\infty} b_{j} u_{t-j}, \quad y_{t}=\sum_{j=0}^{\infty} c_{j} w_{t-j}
$$

where $u_{t}, w_{t}$ are zero mean, independently and identically distributed processes, and the coefficient sequences $\left\{b_{j}\right\}$ and $\left\{c_{j}\right\}$ decay hyperbolically. If $X_{n}$ and $Y_{n}$ denote suitably normalized partial sum processes on the unit interval for a sample of size $n$, it is known under fairly general assumptions that $\left(X_{n}, Y_{n}\right) \stackrel{d}{\rightarrow}(X, Y)$ where the limit processes are fractional Brownian motions, as defined by Mandelbrot and van Ness (1968). For exemplar case $X$, the well-known formula is

$$
X(\xi)=\frac{1}{\Gamma\left(d_{Y}+1\right)}\left[\int_{0}^{\xi}(\xi-\tau)^{d_{X}} d U(\tau)+\int_{-\infty}^{0}\left((\xi-\tau)^{d_{X}}-(-\tau)^{d_{X}}\right) d U(\tau)\right]
$$

where $d_{X}$ is the fractional integration parameter, typically defining the hyperbolic decay rate by $b_{j}=O\left(j^{d_{X}-1}\right)$, and $U$ is regular Brownian motion on $\mathbb{R}$. Fractional noise processes are a well-known simple case, in which

$$
b_{j}=\frac{\Gamma\left(j+d_{X}\right)}{\Gamma\left(d_{X}\right) \Gamma(j+1)} \quad c_{j}=\frac{\Gamma\left(j+d_{Y}\right)}{\Gamma\left(d_{Y}\right) \Gamma(j+1)}
$$

for $-\frac{1}{2}<d_{X}, d_{Y}<\frac{1}{2}$. In this case,

$$
X_{n}(\xi)=n^{-1 / 2-d_{X}} \sum_{t=1}^{[n \xi]} x_{t}, \quad Y_{n}(\xi)=n^{-1 / 2-d_{Y}} \sum_{t=1}^{[n \xi]} y_{t}
$$

for $0 \leq \xi \leq 1$, where $[x]$ denotes the largest integer not exceeding $x$. Considerably greater generality will be permitted, although parameters $d_{X}$ and $d_{Y}$, subject to these constraints, will in all cases index the rate of lag decay. The best general conditions currently known for these results are given by Davidson and de Jong (2000) (henceforth, DDJ).

In this paper, our concern is the limiting distribution of the random variable

$$
G_{n}=\frac{1}{K(n)} \sum_{t=1}^{n-1} \sum_{s=1}^{t} x_{s} y_{t+1}
$$

where $K(n)$ is a function of sample size which, for the case of (1.3) at least, can be set as $n^{1+d_{X}+d_{Y}} \cdot{ }^{1}$ Double sums of the type in (1.5) arise in the theory of cointegration. In the case $x_{t}=y_{t}$ they appear in the formula for the Dickey-Fuller statistic. This distribution was studied by Sowell (1990) who obtained a special form for the distribution analogous to the ordinary unit root case (Phillips 1987). In the multivariate context they arise, for example, in tests of cointegrating rank (Johansen 1991) and cointegrating regressions. For these latter applications, no distribution theory has been available to date.

A weak limit for (1.5) has been derived from the harmonic representation of the variables, whenever these are defined. The analysis of Chan and Terrin (1995) has been extended by Davidson and Hashimzade (2008) (henceforth, DH) to include 'causal' models of the type considered

\footnotetext{
${ }^{1}$ The divergence rates for the mean and mean deviation sequences can differ, and in those cases we define $K(n)$ to normalize $G_{n}-E\left(G_{n}\right)$, whereas $G_{n}$ itself may diverge.
} 
here. This paper explores the counterpart of their solution in the time domain. There are several reasons why this alternative approach provides an essential extension. The general weak convergence proofs given by $\mathrm{DH}$ are restricted to the case $d_{X}+d_{Y}>0$, and the 'standard' case $d_{X}=d_{Y}=0$ is especially intractable, because the harmonic representation of the integral breaks down (with undefined expectation) when the processes have summable covariances. While there is no difficulty in constructing more general dependence models than the fractional noise example given, the harmonic representation requires Gaussian, identically distributed shocks - a restrictive requirement for econometric modelling. Working in the time domain allows all these limitations to be relaxed.

The paper is structured as follows. After specifying our assumptions in Section 2, in Section 3 we decompose $G_{n}$ into components $G_{1 n}, G_{2 n}$ and $G_{3 n}$, where $G_{2 n}$ has a mean square limit and the other two terms have zero means. In Section 4 , heuristic arguments are used to establish the limit distributions of these latter terms as stochastic integrals of Itô type, although with respect to different integrator processes. In Section 5, we demonstrate the weak convergence formally. The implications of our results for cointegrating regression analysis are briefly summarized in the concluding Section 6. Proofs are gathered in the Appendix.

\section{Setup and Assumptions}

The specific assumptions to be adopted are as follows.

Assumption 1 The collection $\left\{u_{t}, w_{t} ; t \in \mathbb{Z}\right\}$ are identically and independently distributed with zero mean and covariance matrix

$$
\mathrm{E}\left[\begin{array}{l}
u_{t} \\
w_{t}
\end{array}\right]\left[\begin{array}{ll}
u_{t} & w_{t}
\end{array}\right]=\boldsymbol{\Omega}=\left[\begin{array}{ll}
\omega_{u u} & \omega_{u w} \\
\omega_{u w} & \omega_{w w}
\end{array}\right]
$$

and $\mu_{u w}^{4}=\mathrm{E}\left(u_{t}^{2} w_{t}^{2}\right)<\infty . u_{t}=w_{t}$ is an admissible case.

These random variables define the filtered probability space on which our processes live, denoted $(\Omega, \mathcal{F}, P, \boldsymbol{F})$ where

$$
\boldsymbol{F}=\left\{\mathcal{F}_{t}, t \in \mathbb{Z} ; \mathcal{F}_{t} \subseteq \mathcal{F} \text { all } t, \text { and } \mathcal{F}_{t} \subseteq \mathcal{F}_{s} \text { iff } t \leq s\right\}
$$

The pair $\left(u_{t}, w_{t}\right)$ is adapted to $\mathcal{F}_{t}$, and in this setup we may also use the notation $\mathcal{F}_{n}(r)=\mathcal{F}_{[n r]}$ for $0 \leq r \leq 1$ where $n$ is sample size. Further, letting $\mathcal{F}(r)$ represent the limiting case as $n \rightarrow \infty$, $(X(r), Y(r))$ are measurable with respect to $\mathcal{F}(r)$ and accordingly will be called $\boldsymbol{F}$-adapted.

Assumption 2 The sequences $\left\{b_{j}\right\}_{0}^{\infty}$ and $\left\{c_{j}\right\}_{0}^{\infty}$ depend, respectively, on parameters $d_{X} \in$ $\left(-\frac{1}{2}, \frac{1}{2}\right)$ and $d_{Y} \in\left(-\frac{1}{2}, \frac{1}{2}\right)$ and sequences $\left\{L_{X}(j)\right\}$ and $\left\{L_{Y}(j)\right\}$ that are at most slowly varying at infinity. These sequences satisfy one of the following conditions, stated for $\left\{b_{j}\right\}_{0}^{\infty}$ as exemplar case:

(a) If $0<d_{X}<\frac{1}{2}$ then $b_{j}=\Gamma\left(d_{X}\right)^{-1}(j+1)^{d_{X}-1} L_{X}(j)$.

(b) If $d_{X}=0$ then $0<\left|\sum_{j=0}^{\infty} b_{j}\right|<\infty$, and $b_{j}=O\left(j^{-1-\delta}\right)$ for $\delta>0$.

(c) If $-\frac{1}{2}<d_{X}<0$ then $b_{0}=a_{0}$ and $b_{j}=a_{j}-a_{j-1}$ for $j>0$ where $a_{j}=\Gamma\left(1+d_{X}\right)^{-1}(j+$ $1)^{d_{X}} L_{X}(j)$. 
Under these assumptions, we set $K(n)=n^{1+d_{X}+d_{Y}} L_{X}(n) L_{Y}(n)$ in (1.5). While the 'pure fractional' cases represented by (1.3) satisfy Assumption 2, the assumption only controls the tail behaviour of the sequences, and allows arbitrary forms for a finite number of the lag coefficients. In particular, the $x_{t}$ and $y_{t}$ processes may be stable invertible $\operatorname{ARFIMA}(p, d, q)$ processes. Suppose more generally that $x_{t}=(1-L)^{-d_{X}} \theta(L) u_{t}$ where $\theta(L)$ is, in general, a power series in the lag operator with absolutely summable coefficients such that $\theta_{j}=O\left(j^{-1-\delta}\right)$ for $\delta>0$. Letting for $d_{X}>0$ the identity $a(L)=(1-L)^{-d_{X}}$ define the coefficients $a_{j}$, such that $a_{j} \sim \Gamma\left(d_{X}\right)^{-1} j^{d_{X}-1},{ }^{2}$ note the following result.

Proposition 2.1 The sequence $\left\{b_{j}\right\}$ defined by $b(L)=a(L) \theta(L)$ satisfies $b_{j} \sim \theta(1) \Gamma\left(d_{X}\right)^{-1} j^{d_{X}-1}$ as $j \rightarrow \infty$.

(All proofs are given in the Appendix.) The slowly varying component can be defined to represent the ratio of $b_{j}$ to the approximating sequence. Also, since $\Omega$ is unrestricted, we could impose the normalization $\theta(1)=1$, if desired, with no loss of generality.

Assumption 2(b) rules out the non-summable case $\delta=0$, where the sum of the coefficients diverges logarithmically. This is to avoid large complications of doubtful relevance. Be careful to note that $\delta$ is not a fractional differencing coefficient in this case. The case $d_{X}<0$ under Assumption 2(c) has the 'overdifferenced' property, implying in particular that $\left|\sum_{k=0}^{j} b_{k}\right|=$ $O\left(j^{d_{X}}\right)$. In the pure fractional model, represented by (1.3), note that $b_{0}=1$ and $b_{j}=0$ for $j>0$ in the case $d_{X}=0$, whereas $b_{j}<0$ for all $j>0$ if $d_{X}<0$.

In applications, our pair of processes would typically be embedded in a vector Wold representation of the VARFIMA type, such as

$$
\left[\begin{array}{c}
x_{t} \\
y_{t}
\end{array}\right]=\left[\begin{array}{cc}
(1-L)^{-d_{X}} & 0 \\
0 & (1-L)^{-d_{Y}}
\end{array}\right]\left[\begin{array}{cc}
\theta_{X X}(L) & \theta_{X Y}(L) \\
\theta_{Y X}(L) & \theta_{Y Y}(L)
\end{array}\right]\left[\begin{array}{c}
u_{t} \\
w_{t}
\end{array}\right]
$$

To show the limit distribution of product moments in this framework is a simple application of the continuous mapping theorem to the results explored in this paper. Note how $x_{t}$ and $y_{t}$ are the sums of terms of the type (1.1), involving $\left\{u_{t}\right\}$ and $\left\{w_{t}\right\}$ respectively, so (1.5) becomes a sum of four terms involving respectively the driving pairs $\left\{u_{t}, u_{t}\right\},\left\{u_{t}, w_{t}\right\},\left\{w_{t}, u_{t}\right\}$ and $\left\{w_{t}, w_{t}\right\}$. Our analysis can be applied to each of these cases in turn, with suitable redefinition of symbols.

A standard application of our results will be to models of fractional cointegration. In this case, we may conjecture the existence of a relationship between nonstationary variables $p_{t}=\sum_{s=1}^{t} x_{t}$ and $q_{t}=\beta p_{t}+y_{t}$, for a cointegrating parameter $\beta$. In the case where $d_{Y}>0$ we have a case of so-called fractional cointegration, in which the cointegrating residual exhibits long memory. ${ }^{3}$ Least squares estimation of this relation yields

$$
\hat{\beta}=\beta+\frac{\sum_{t=1}^{n} p_{t} y_{t}}{\sum_{t=1}^{n} p_{t}^{2}}
$$

where the numerator of the error-of-estimate is an object of the form (1.5) apart from normalization. Until now, little has been known about the distribution of random variables of this form, subject to Assumptions 1 and 2. One question of particular interest must be the possible

\footnotetext{
${ }^{2}$ The symbol ' $\sim$ ' here denotes that the ratio of the connected sequences converges to 1 as $j \rightarrow \infty$.

${ }^{3}$ This phenomenon appears far from unusual in applied studies. As a well-known example, Lettau and Ludvigson (2001) report a cointegrating vector for quarterly US consumption, labour income and household asset wealth,19522001. The data are published on the second author's web page. Log-periodogram regression performed on their estimated vector yields a fractional integration parameter $(d)$ in excess of 0.8 ; significantly under 1 but nonetheless in the covariance non-stationary range, a case outside even the range of possibilities being entertained here. See Davidson (2006) for further details.
} 
existence of transformations to mixed normality, allowing standard inference procedures for such models. Following the presentation of our main results, we return to this issue in Section 6 .

\section{Some Properties of $G_{n}$}

Expand (1.5), by substitution from (1.1), as

$$
G_{n}=\frac{1}{K(n)} \sum_{t=1}^{n-1} \sum_{s=1}^{t} \sum_{j=0}^{\infty} \sum_{k=0}^{\infty} b_{k} c_{j} u_{s-k} w_{t+1-j} .
$$

Then decompose this sum as $G_{n}=G_{1 n}+G_{2 n}+G_{3 n}$ where

$$
\begin{aligned}
& G_{1 n}=\frac{1}{K(n)} \sum_{t=1}^{n-1} \sum_{s=1}^{t} \sum_{k=0}^{\infty} \sum_{j=0}^{k+t-s} b_{k} c_{j} u_{s-k} w_{t+1-j} \\
&=\frac{1}{K(n)} \sum_{t=1}^{n-1} \sum_{s=1}^{t} \sum_{j=0}^{\infty} \sum_{k=\max \{0, j+s-t\}}^{\infty} b_{k} c_{j} u_{s-k} w_{t+1-j} \\
& G_{2 n}=\frac{1}{K(n)} \sum_{t=1}^{n-1} \sum_{s=1}^{t} \sum_{k=0}^{\infty} b_{k} c_{k+t-s+1} u_{s-k} w_{s-k}
\end{aligned}
$$

and

$$
G_{3 n}=\frac{1}{K(n)} \sum_{t=1}^{n-1} \sum_{s=1}^{t} \sum_{k=0}^{\infty} \sum_{j=k+t-s+2}^{\infty} b_{k} c_{j} u_{s-k} w_{t+1-j} .
$$

Thus, $G_{1 n}$ contains those terms, and only those terms, in which $s-k \leqslant t-j$, so that the time indices of $w$ strictly exceed those of $u$, and hence $\mathrm{E}\left(G_{1 n}\right)=0$. In $G_{2 n}, s-k=t+1-j$ such that the time indices of $u$ and $w$ match. In $G_{3 n}, s-k>t+1-j$ such that the indices of $u$ lead those of $w$, and $\mathrm{E}\left(G_{3 n}\right)=0$.

The properties of $G_{2 n}$ depend on the sign of $d_{X}+d_{Y}$, and we consider the various cases in turn.

Proposition 3.1 If $d_{X}+d_{Y}>0$ then $\mathrm{E}\left(G_{2 n}\right) \rightarrow \lambda_{X Y}$ where

$$
\begin{aligned}
\lambda_{X Y}=\frac{\omega_{u w}}{\Gamma\left(d_{X}+1\right) \Gamma\left(d_{Y}+1\right)\left(d_{X}+d_{Y}\right)}\left(\frac{d_{Y}}{\left(1+d_{X}+d_{Y}\right)}+\right. \\
\left.\int_{0}^{\infty}\left[d_{Y}(1+\tau)^{d_{X}+d_{Y}}+d_{X} \tau^{d_{X}+d_{Y}}-\left(d_{X}+d_{Y}\right)(1+\tau)^{d_{Y}} \tau^{d_{X}}\right] d \tau\right) .
\end{aligned}
$$

Letting $\lambda_{Y X}$ denote the same limit with $x_{t}$ and $y_{t}$ interchanged, also note that

$$
\begin{aligned}
\lambda_{X Y}+\lambda_{Y X}= & \frac{\omega_{u w}}{\Gamma\left(d_{X}+1\right) \Gamma\left(d_{Y}+1\right)} \times \\
& \left(\frac{1}{\left(1+d_{X}+d_{Y}\right)}+\int_{0}^{\infty}\left((1+\tau)^{d_{X}}-\tau^{d_{X}}\right)\left((1+\tau)^{d_{Y}}-\tau^{d_{Y}}\right) d \tau\right) \\
= & \psi_{X Y}
\end{aligned}
$$

where

$$
\psi_{X Y}=\lim _{n \rightarrow \infty} \frac{1}{K(n)} \mathrm{E}\left(\sum_{t=1}^{n} x_{t} \sum_{t=1}^{n} y_{t}\right)
$$


This is the off-diagonal element of $\boldsymbol{\Psi}$, the long-run covariance matrix of the processes, according to equation (3.12) of DDJ. Considering the decomposition

$$
\mathrm{E}\left(\sum_{t=1}^{n} x_{t} \sum_{t=1}^{n} y_{t}\right)=\sum_{t=1}^{n} \mathrm{E}\left(x_{t} y_{t}\right)+\sum_{t=1}^{n-1} \sum_{s=1}^{t} \mathrm{E}\left(x_{s} y_{t+1}\right)+\sum_{t=1}^{n-1} \sum_{s=1}^{t} \mathrm{E}\left(y_{s} x_{t+1}\right)
$$

where the second term on the right corresponds to $K(n) \mathrm{E}\left(G_{n}\right)$, note that

$$
\mathrm{E}\left(x_{t} y_{t}\right)=\sigma_{X Y}=\omega_{u w} \sum_{j=0}^{\infty} b_{j} c_{j}<\infty .
$$

The first right-hand side term in (3.7) is $O(n)$, and hence this term is of small order under the normalization $K(n)$. The other two terms converge to $\lambda_{X Y}$ and $\lambda_{Y X}$ respectively under the same normalization, as indicated by (3.5). Observe that $\lambda_{X Y}$ depends only on $d_{X}, d_{Y}$ and $\omega_{u w}$ since any short-run parameters have been absorbed into the functions $L_{X}$ and $L_{Y}$; compare Proposition 2.1 for example. The sign of $\lambda_{X Y}$ matches that of $d_{Y}$, and if $d_{Y}=0$, then $\lambda_{X Y}=0$. When $d_{X}>0$, the cases where $y_{t}$ is i.i.d. $\left(c_{j}=0\right.$ for $\left.j>0\right)$ and is merely weakly dependent $\left(d_{Y}=0\right)$, are equivalent asymptotically.

We give these results in the easily interpretable form of (3.4) but for computational purposes the following closed-form expression is more useful.

Proposition 3.2 If $d_{X}+d_{Y}>0, \lambda_{X Y}=\frac{\omega_{u w} \Gamma\left(1-d_{X}-d_{Y}\right)}{\pi\left(1+d_{X}+d_{Y}\right)\left(d_{X}+d_{Y}\right)} \sin \pi d_{Y}$.

This formula matches that obtained for the causal model by DH (page 268), indicating that the harmonic and moving average approaches to constructing fractional processes yield equivalent results, at least in mean. The closed form of (3.5)

$$
\psi_{X Y}=\frac{\omega_{u w} \Gamma\left(1-d_{X}-d_{Y}\right)}{\left(1+d_{X}+d_{Y}\right)}\left(\frac{\sin \pi d_{Y}+\sin \pi d_{X}}{\pi\left(d_{Y}+d_{X}\right)}\right)
$$

follows directly.

Next consider the cases where $d_{X}+d_{Y}$ is zero or negative. In the latter case, $\mathrm{E}\left(G_{2 n}\right)$ diverges.

Proposition 3.3 If $d_{X}+d_{Y} \leq 0$ and $\omega_{w u} \neq 0$, then $\mathrm{E}\left(G_{2 n}\right)=O(n / K(n))$.

In this instance there is no decomposition of $\psi_{Y X}$ into components of the form $\lambda_{X Y}$, and the three terms in (3.7) are each of $O(n)$. We may write $n^{-1} \sum_{t=1}^{n} \mathrm{E}\left(x_{t} y_{t}\right)=\sigma_{X Y}$ and also

$$
\begin{aligned}
\frac{1}{n} \sum_{t=1}^{n-1} \sum_{s=1}^{t} \mathrm{E}\left(x_{s} y_{t+1}\right) & \rightarrow \lambda_{X Y}^{*} \\
\frac{1}{n} \sum_{t=1}^{n-1} \sum_{s=1}^{t} \mathrm{E}\left(y_{s} x_{t+1}\right) & \rightarrow \lambda_{Y X}^{*} .
\end{aligned}
$$

These limits are finite constants depending on summable sequences of weights, hence necessarily different from $\lambda_{X Y}$ and $\lambda_{Y X}$. Note that $\mathrm{E}\left(\sum_{t=1}^{n} x_{t}\right)^{2}=O\left(n^{2 d_{X}+1}\right)$ and $\mathrm{E}\left(\sum_{t=1}^{n} y_{t}\right)^{2}=O\left(n^{2 d_{Y}+1}\right)$ (compare DDJ Lemmas 3.1 and 3.3). For $d_{X}+d_{Y}<0$ the left-hand side of (3.7) is therefore necessarily $o(n)$, by the Cauchy-Schwarz inequality, and so $\sigma_{X Y}+\lambda_{X Y}^{*}+\lambda_{Y X}^{*}=0$. Formula (3.9) is nonetheless well defined for $d_{X}+d_{Y} \leq 0$. Under the normalization $n$ the covariance vanishes, but under normalization $K(n)$ the limit in (3.6) is well-defined and equal to (3.5) (equivalently, 
to (3.9)) as shown in DDJ Lemma 3.3. These conclusions assume $\omega_{u w} \neq 0$, but if $u_{t}$ and $w_{t}$ are contemporaneously uncorrelated, implying under Assumption 1 that the cross-correlogram is zero at all orders, then each of the terms in (3.7) is zero identically. Then (3.5) holds trivially whatever the sign of $d_{X}+d_{Y}$, since $\lambda_{X Y}=\lambda_{Y X}=0$.

The following result shows that $G_{2 n}$ is a consistent estimator of the mean, albeit not a feasible one. Let ' $\stackrel{L_{2}}{\rightarrow}$ denote convergence in mean square.

Theorem 3.1 If Assumptions 1 and 2 hold, $G_{2 n}-\mathrm{E}\left(G_{2 n}\right) \stackrel{L_{2}}{\rightarrow} 0$.

The important implication is that the limit distribution of $G_{1 n}+G_{3 n}$ matches that of the mean deviation of $G_{n}$, not forgetting that the mean diverges under the given normalization when $d_{X}+d_{Y}<0$.

One further result concerning the behaviour of the contemporaneous covariance term is generally needed for the analysis of regression models. Let ' $\stackrel{d}{\rightarrow}$ ' denote convergence in distribution.

Theorem 3.2 Let Assumptions 1 and 2 hold. Then

(i) $n^{-1} \sum_{t=1}^{n} x_{t} y_{t} \stackrel{L_{2}}{\rightarrow} \sigma_{X Y}$.

(ii) If $\omega_{u w}=0,-\frac{1}{2}<d_{Y} \leq 0$ and $-\frac{1}{2}<d_{X} \leq 0$, then $n^{-1 / 2} \sum_{t=1}^{n} x_{t} y_{t} \stackrel{d}{\rightarrow} N(0, V)$ where $V<\infty$.

\section{Stochastic Integrals}

In this section we use heuristic arguments to construct limiting forms for the terms $G_{1 n}$ and $G_{3 n}$, to be denoted by $\Xi_{1, X Y}$ and $\Xi_{3, X Y}$ respectively. Letting $\Xi_{X Y}=\Xi_{1, X Y}+\Xi_{3, X Y}$, we subsequently show that $G_{n}-\mathrm{E}\left(G_{n}\right) \stackrel{d}{\rightarrow} \Xi_{X Y}$

Consider $G_{1 n}$ first. Replacing the summation over $j$ in (3.1) by the summation over $m=t-j$, and the summation over $k$ by the summation over $i=s-k$, rewrite $G_{1 n}$ as

$$
\begin{aligned}
G_{1 n} & =\frac{1}{K(n)} \sum_{t=1}^{n-1} \sum_{s=1}^{t} \sum_{m=-\infty}^{t} \sum_{i=-\infty}^{\min \{s, m\}} b_{s-i} c_{t-m} u_{i} w_{m+1} \\
& =\frac{1}{K(n)} \sum_{m=-\infty}^{n-1} w_{m+1} \sum_{i=-\infty}^{m} u_{i} \sum_{t=\max \{1, m\}}^{n-1} c_{t-m}\left(\sum_{s=\max \{1, i\}}^{t} b_{s-i}\right) \\
& =\frac{1}{n} \sum_{m=-\infty}^{n-1} q_{n m} w_{m+1}
\end{aligned}
$$

where $q_{n m}=\sum_{i=-\infty}^{m} a_{n i m} u_{i}$ and

$$
a_{\text {nim }}=\frac{n}{K(n)} \sum_{t=\max \{1-m, 0\}}^{n-1-m} c_{t}\left(\sum_{s=\max \{1-i, 0\}}^{t+m-i} b_{s}\right)
$$

Lemma 4.1 For real-valued indices $r, p$ with $-\infty<p \leq r \leq 1, a_{n[n p][n r]}=A_{X Y}(r, p)+o(1)$ as $n \rightarrow \infty$ where, letting $1_{\{.\}}$denote the indicator of its argument,

$$
A_{X Y}(r, p)=\frac{(r-p)^{d_{X}}(1-r)^{d_{Y}} F\left(-d_{X}, d_{Y}, 1+d_{Y} ;-\frac{1-r}{r-p}\right)}{\Gamma\left(1+d_{X}\right) \Gamma\left(1+d_{Y}\right)}
$$




$$
\begin{array}{r}
-1_{\{r<0\}} \frac{(r-p)^{d_{X}}(-r)^{d_{Y}} F\left(-d_{X}, d_{Y}, 1+d_{Y} ;-\frac{-r}{r-p}\right)}{\Gamma\left(1+d_{X}\right) \Gamma\left(1+d_{Y}\right)} \\
-1_{\{p<0\}} \frac{(-p)^{d_{X}}\left((1-r)^{d_{Y}}-1_{\{r<0\}}(-r)^{d_{Y}}\right)}{\Gamma\left(1+d_{X}\right) \Gamma\left(1+d_{Y}\right)}
\end{array}
$$

and

$$
F(a, b, c ; z)=\frac{\Gamma(c)}{\Gamma(a) \Gamma(b)} \sum_{j=0}^{\infty} \frac{\Gamma(a+j) \Gamma(b+j)}{\Gamma(c+j) j !}(-z)^{j}
$$

represents the hypergeometric function.

Making the substitutions $d U(p)$ for $u_{[n p]} / \sqrt{n}$ and $d W(r)$ for $w_{[n r]} / \sqrt{n}$, the limit of the random variable in (4.1) can be expressed heuristically in the form $\Xi_{1, X Y}=\int_{-\infty}^{1} Q(r) d W(r)$ where $Q(r)=\int_{-\infty}^{r} A_{X Y}(r, p) d U(p)$. Note that when $d_{Y}=0, Q(r)=X(r)$ for $r \geq 0$ and 0 for $r<0$, and $\Xi_{1, X Y}$ reduces to the Itô integral of a fractional Brownian motion integrand, as analysed in DDJ. In the general case, we ought to remark on the potential existence issue posed by a functional of Brownian motion with infinitely remote starting point. We shall show in the sequel that these integrals can be constructed as the mean-square limits of integrals on the finite intervals $[-N, r]$ and $[-N, 1]$, respectively, as $N \rightarrow \infty$. Of course, the fractional Brownian motion (1.2) itself is well-defined on just the same basis.

Next, consider $G_{3 n}$. Proceeding in the same way as before, setting $m=t+1-j$ and $i=s-k$, we obtain from $(3.3)$

$$
\begin{aligned}
G_{3 n} & =\frac{1}{K(n)} \sum_{t=1}^{n-1} \sum_{s=1}^{t} \sum_{k=0}^{\infty} \sum_{j=k+t-s+2}^{\infty} b_{k} c_{j} u_{s-k} w_{t+1-j} \\
& =\frac{1}{K(n)} \sum_{i=-\infty}^{n-1} u_{i} \sum_{m=-\infty}^{i-1} w_{m} \sum_{s=\max \{i, 1\}}^{n-1} b_{s-i} \sum_{t=s}^{n-1} c_{t+1-m} \\
& =\frac{1}{K(n)} \sum_{i=-\infty}^{n-1} h_{i-1} u_{i}
\end{aligned}
$$

where $h_{i}=\sum_{m=-\infty}^{i} e_{n m i} w_{m}$ and

$$
e_{n m i}=\frac{n}{K(n)} \sum_{s=\max \{0,1-i\}}^{n-1-i} b_{s}\left(\sum_{t=s+i+1-m}^{n-m} c_{t}\right) .
$$

Lemma 4.2 For real-valued indices $r, p$ with $-\infty<r \leq p \leq 1, e_{n[n r][n p]}=E_{X Y}(p, r)+o(1)$ as $n \rightarrow \infty$, where

$$
\begin{aligned}
E_{X Y}(p, r)= & \frac{1}{\Gamma\left(d_{Y}+1\right) \Gamma\left(d_{X}+1\right)} \\
& \times\left[(1-p)^{d_{X}}\left((1-r)^{d_{Y}}-(p-r)^{d_{Y}} F\left(-d_{Y}, d_{X} ; 1+d_{X} ;-\frac{1-p}{p-r}\right)\right)\right. \\
& \left.\quad-1_{\{p<0\}}(-p)^{d_{X}}\left((1-r)^{d_{Y}}-(p-r)^{d_{Y}} F\left(-d_{Y}, d_{X} ; 1+d_{X} ; \frac{-p}{p-r}\right)\right)\right] .
\end{aligned}
$$


This construction closely parallels the one in Lemma 4.1 except that in this case $p \geq r$. It allows us to express the limit in the form $\Xi_{3, X Y}=\int_{-\infty}^{1} H(p) d U(p)$ where $H(p)=\int_{-\infty}^{p} E_{X Y}(p, r) d W(r)$. Observe that $E_{X Y}(p, r)=0$ for all $p$ and $r$ when $d_{Y}=0$, so that this term arises only in the case of fractional integrator functions.

Notice the important fact that both $\Xi_{1, X Y}$ and $\Xi_{3, X Y}$ are stochastic integrals of $\boldsymbol{F}$-adapted Gaussian integrand processes with respect to $\boldsymbol{F}$-adapted Brownian motions. Therefore, these integrals are of Itô type. Subject to sufficient regularity conditions on the integrands, essentially those of finite variances and almost sure continuity, plus the validity of mean-squared approximations by integrals with finite domain of integration, they may be analysed in the conventional fashion. Section 5 provides the requisite results.

Under assumptions such that both convergence results hold, ${ }^{4}$ it appears natural to equate the random variable $\Xi_{X Y}+\lambda_{X Y}$ with the stochastic integral $\int_{0}^{1} X d Y$ as defined (with appropriate changes of notation) in DH Theorem 4.1. We have shown in Lemma 3.2 that the means match. To validate the representation as an integral, however, we also need to establish that the formulae satisfy the integration by parts rule. In DH Corollary 4.1, this was shown to hold in expectation for the harmonic representation. Here, we can go further and show the following result, which does not depend on parameter sign restrictions.

Proposition 4.1 $\Xi_{X Y}+\Xi_{Y X}+\psi_{X Y}=X(1) Y(1)$.

\section{Weak Convergence}

Building on the results in Section 3 on the behaviour of the mean sequence, the general result to be established in this section is the following.

Proposition 5.1 Let Assumptions 1 and 2 hold.

(i) If $d_{X}+d_{Y}>0$, then $G_{n} \stackrel{d}{\rightarrow} \Xi_{X Y}+\lambda_{X Y}$.

(ii) If $d_{X}+d_{Y}=0$, then $G_{n} \stackrel{d}{\rightarrow} \Xi_{X Y}+\lambda_{X Y}^{*}$.

(iii) If $d_{X}+d_{Y}<0$ and $\lambda_{X Y}^{*} \neq 0$, then $\frac{K(n)}{n} G_{n} \stackrel{L_{2}}{\rightarrow} \lambda_{X Y}^{*}$.

(iv) If $d_{X}+d_{Y}<0$ and $\lambda_{X Y}^{*}=0$, then $G_{n} \stackrel{d}{\rightarrow} \Xi_{X Y}$.

Note that case (iii) has already been established in Theorem 3.1, subject to the components $G_{1 n}$ and $G_{3 n}$ being $O_{p}(1)$ while $G_{2 n}=O_{p}(n / K(n))$.

Define cadlag processes $X_{n}=n^{-1 / 2-d_{X}} L_{X}(n)^{-1} \sum_{t=1}^{n} x_{t}$ and $Y_{n}=n^{-1 / 2-d_{Y}} L_{Y}(n)^{-1} \sum_{t=1}^{n} y_{t}$. Then, Proposition 5.1 will follow from Propositions 3.1 and 3.3 and Theorem 3.1 in combination with the following result, which is the main concern of this section.

Theorem 5.1 Under Assumptions 1 and 2,

$$
\left(X_{n}, Y_{n}, G_{n}-\mathrm{E}\left(G_{n}\right)\right) \stackrel{d}{\rightarrow}\left(X, Y, \Xi_{X Y}\right)
$$

where $\Xi_{X Y}=\Xi_{1, X Y}+\Xi_{3, X Y}$, and $\stackrel{d}{\rightarrow}$ denotes joint weak convergence in $D_{\mathbb{R}^{2}}[0,1] \times \mathbb{R}$ where $D_{\mathbb{R}^{2}}[0,1]$ denotes the space of cadlag pairs equipped with the Skorokhod topology.

\footnotetext{
${ }^{4}$ Specifically, we require $d_{X} \geq 0$ and $d_{Y} \geq 0$ with at least one strict inequality.
} 
The result for the first two members of (5.1) is shown in DDJ. Since the limit processes are almost surely continuous, it is sufficient for joint convergence that arbitrary linear combinations of $\left(X_{n}, Y_{n}, G_{n}-\mathrm{E}\left(G_{n}\right)\right)$ converge to the corresponding combinations of the limit processes (see Davidson 1994, Theorem 29.16). Since the process elements are all defined with respect to the same filtration, these requirements follow directly. In practice, we show $\left(X_{n}, Y_{n}, G_{1 n}, G_{3 n}\right) \stackrel{d}{\rightarrow}$ $\left(X, Y, \Xi_{1, X Y}, \Xi_{3, X Y}\right)$ where the limit random variables $\Xi_{1, X Y}$ and $\Xi_{3, X Y}$ can be identified with the Itô integrals on the intervals $(-\infty, 1]$. The continuous mapping theorem then yields Theorem 5.1 .

A further rearrangement of (4.1) yields

$$
G_{1 n}=\frac{1}{n} \sum_{m=-N n}^{n-1} q_{n m}^{N} w_{m+1}+\frac{1}{n} \sum_{m=-N n}^{n-1}\left(q_{n m}-q_{n m}^{N}\right) w_{m+1}+\frac{1}{n} \sum_{m=-\infty}^{-N n-1} q_{n m} w_{m+1}
$$

where $q_{n m}^{N}=\sum_{i=-N n}^{m} a_{n i m} u_{i}, a_{n i m}$ is defined in (4.2) and $N>0$ is a fixed value to be chosen. In the same way, write

$$
G_{3 n}=\frac{1}{n} \sum_{i=-N n}^{n-1} h_{n, i-1}^{N} u_{i}+\frac{1}{n} \sum_{i=-N n}^{n-1}\left(h_{n, i-1}-h_{n, i-1}^{N}\right) u_{i}+\frac{1}{n} \sum_{i=-\infty}^{-N n-1} h_{n, i-1} u_{i}
$$

where $h_{n i}^{N}=\sum_{m=-N n}^{i} e_{n m i} w_{m}$. The strategy of proof of Theorem 5.1 suggested by these decompositions involves three steps, which we describe for $G_{1 n}$ as the exemplar case.

1. Define the cadlag arrays

$$
Q_{n}^{N}(r)=\frac{1}{\sqrt{n}} \sum_{i=-N n}^{[n r]} a_{n i[n r]} u_{i}, \quad W_{n}^{N}(r)=\frac{1}{\sqrt{n}} \sum_{m=-N n}^{[n r]} w_{m}
$$

and show that $Q_{n}^{N} \stackrel{d}{\rightarrow} Q^{N}$, an almost surely continuous Gaussian process on the interval $[-N, 1]$. Also, by standard arguments, $W_{n}^{N} \stackrel{d}{\rightarrow} W^{N}$ where $W^{N}$ is a Brownian motion on the interval $[-N, 1]$. Since $q_{n, m-1}^{N}$ is a linear process in i.i.d. shocks, by Assumption 1, Step 1 can be tackled by a minor extension of Theorem 3.1 of de Jong and Davidson (2000) (henceforth, DJD).

2. $\left(Q^{N}, W^{N}\right)$ are adapted to a common filtration $\boldsymbol{F}$ defined in (2.2), with respect to which $W^{N}$ is a martingale. We therefore deduce by standard arguments that

$$
\left(Q_{n}^{N}, W_{n}^{N}, \frac{1}{n} \sum_{m=-N n}^{n} q_{n, m-1}^{N} w_{m}\right) \stackrel{d}{\rightarrow}\left(Q^{N}, W^{N}, \int_{-N}^{1} Q^{N} d W^{N}\right) .
$$

3. Show that by taking $N$ large enough, the second and third terms of (5.2) can be made as small as desired in $L_{2}$ norm, allowing the limit random process to be formally represented as $\Xi_{1, X Y}=\int_{-\infty}^{1} Q d W$.

The arguments to establish the validity of these steps are given for the case of $G_{1 n}$ in Section 5.1. The case of $G_{3 n}$ is on similar lines, replacing $a$ by $e, A$ by $E, Q$ by $H$, and exchanging $w, u$ and $W, U$ in formulae. These results are given in Section 5.2. 


\subsection{The Case of $G_{1 n}$}

We use Lemma 4.1 to show the following properties, invoking Assumptions 1 and 2 in each case.

Lemma 5.1 Let $v_{n m}^{a}=n^{-1} \sum_{i=-\infty}^{m} a_{n i m}^{2}$ for $m \in(-\infty, n)$.

(i) $\lim \sup _{n} v_{n,[n r]}^{a}<\infty$ for each fixed $r \in(-\infty, 1]$.

(ii) $\lim \sup _{n} v_{n,[n r]}^{a}=O\left((-r)^{2 d_{Y}+2 d_{X}-3}\right)$ as $r \rightarrow-\infty$.

Lemma $5.2 \sup _{r \in(-\infty, 1]} \limsup _{n} n^{-1} \sum_{i=[n r]+1}^{[n(r+\delta)]} a_{n i[n(r+\delta)]}^{2}=O\left(\delta^{\min \left\{1,2 d_{X}+1\right.}\right)$.

Lemma 5.3 $\sup _{r \in(-\infty, 1]} \limsup _{n} n^{-1} \sum_{i=-\infty}^{[n r]}\left(a_{n i[n(r+\delta)]}-a_{n i[n r]}\right)^{2}=O\left(\delta^{2 d_{X}+1}\right)$.

Step 1 is then implemented by means of the following result.

Theorem $5.2\left(Q_{n}^{N}, W_{n}^{N}\right) \rightarrow\left(Q^{N}, W^{N}\right)$ where $\stackrel{d}{\rightarrow}$ denotes weak convergence in the space of cadlag functions $D_{\mathbb{R}^{2}}[-N, 1]$ endowed with the Skorokhod topology, and $\left(Q^{N}, W^{N}\right)$ are elements of $C_{\mathbb{R}^{2}}[-N, 1]$ a.s..

Be careful to note that the topological space $D_{\mathbb{R}^{2}}[-N, 1]$ is different from $D_{\mathbb{R}}[-N, 1] \times D_{\mathbb{R}}[-N, 1]$. In the former case, the jump times are assumed to be synchronized in the component spaces so that the Skorokhod distances can be defined in terms of a common change-of-time function, while in the latter case they are not. Since the jumps are always the result of discrete observation dates in our applications, the jump times match by default, and there is no problem about satisfying this requirement in practice.

Given these results, we can proceed directly to Step 2, as follows.

Theorem 5.3 The convergence in (5.3) holds where $\stackrel{d}{\rightarrow}$ denotes weak convergence in the space $D_{\mathbb{R}^{2}}[-N, 1] \times \mathbb{R}$ endowed with the Skorokhod topology.

Theorem 5.3 is a special case of Theorem 2.2 of Kurtz and Protter (1991), see also Theorem $7.4^{5}$ of Kurtz and Protter (1995). These results are given for stochastic processes $I$ on $[0, \infty)$ defined by $I(\xi)=\int_{0}^{\xi} H(r) d X(r)$, where $H$ is $\boldsymbol{F}$-adapted and left-continuous, and $X$ is a $\boldsymbol{F}$-semimartingale satisfying a condition of uniformly controlled variations (UCV). This latter condition is directly satisfied by $W_{n}^{N}$ since this is a partial sum of independent and identically distributed shocks with finite variance, and our processes are defined on a compact interval. There is no difficulty about considering the interval $[0, N+1]$, and then re-locating the initial date from 0 to $-N$.

We cannot apply the Kurtz-Protter results in full generality without modification, because in our case the integrands correspond to a family of functionals $Q^{N}(r, \xi)$, and $\int_{-N}^{\xi} Q^{N}(r, \xi) d W(r)$ does not have the form of $I(\xi)$. However, replacing $Q^{N}(r, \xi)$ by $Q^{N}(r, 1)$ defines an integrand process in the appropriate class, and then extracting the pointwise implication for the case $\xi=1$ yields the desired distribution. Since $Q^{N}$ is a.s. continuous according to Lemma 5.2, there is no problem in meeting the left-continuity requirement.

Moving on to Step 3, we show the limiting negligibility of the remainders as follows.

Theorem 5.4 If Assumptions 1 and 2 hold,

\footnotetext{
${ }^{5}$ This theorem is numbered 34 in an alternate version of these notes posted on the internet.
} 
(i) $\lim _{n \rightarrow \infty} \mathrm{E}\left(\frac{1}{n} \sum_{m=-N n}^{n-1}\left(q_{n m}-q_{n m}^{N}\right) w_{m+1}\right)^{2}=O\left(N^{d_{X}+d_{Y}-2}\right)$

(ii) $\lim _{n \rightarrow \infty} \mathrm{E}\left(\frac{1}{n} \sum_{m=-\infty}^{-N n-1} q_{n m} w_{m+1}\right)^{2}=O\left(N^{d_{X}+d_{Y}-3}\right)$.

\subsection{The Case of $G_{3 n}$}

In this section the arguments are effectively the same as those in Section 5.1, although the results differ in formulae and in the details of proofs. We simply state the counterpart results, in abbreviated form where appropriate. The proofs of these results based on the representation in Lemma 4.2, are treated jointly with those of Section 5.1 in the Appendix.

Lemma 5.4 Let $v_{n i}^{e}=n^{-1} \sum_{m=-\infty}^{i} e_{n m i}^{2}$ for $i \in(-\infty, n)$. Then,

(i) $\lim \sup _{n} v_{n,[n p]}^{e}<\infty$ for each fixed $p \in(-\infty, 1]$.

(ii) $\lim \sup _{n} v_{n,[n p]}^{e}=O\left((-p)^{2 d_{Y}+2 d_{X}-3}\right)$ as $p \rightarrow-\infty$.

Lemma 5.5 $\sup _{p \in(-\infty, 1]} \limsup _{n} n^{-1} \sum_{m=[n p]+1}^{[n(p+\delta)]} e_{n m[n(p+\delta)]}^{2}=O\left(\delta^{\min \left\{1,2 d_{X}+1\right\}}\right)$.

Lemma 5.6 $\sup _{p \in(-\infty, 1]} \limsup _{n} n^{-1} \sum_{m=-\infty}^{[n p]}\left(e_{n m[n(p+\delta)]}-e_{n m[n p]}\right)^{2}=O\left(\delta^{2 d_{X}+1}\right)$.

Theorem $5.5\left(H_{n}^{N}, U_{n}^{N}\right) \stackrel{d}{\rightarrow}\left(H^{N}, U^{N}\right) \in C_{\mathbb{R}^{2}}[-N, 1]$ a.s.

Theorem 5.6 $\left(H_{n}^{N}, U_{n}^{N}, \frac{1}{n} \sum_{m=-N n}^{n} u_{m} h_{n, m-1}^{N}\right) \stackrel{d}{\rightarrow}\left(H^{N}, U^{N}, \int_{-N}^{1} H^{N} d U^{N}\right)$.

\section{Theorem 5.7}

(i) $\lim _{n \rightarrow \infty} \mathrm{E}\left(\frac{1}{n} \sum_{m=-N n}^{n-1}\left(h_{n m}-h_{n m}^{N}\right) u_{m+1}\right)^{2}=O\left(N^{d_{X}+d_{Y}-2}\right)$

(ii) $\lim _{n \rightarrow \infty} \mathrm{E}\left(\frac{1}{n} \sum_{m=-\infty}^{-N n-1} h_{n m} u_{m+1}\right)^{2}=O\left(N^{d_{X}+d_{Y}-3}\right)$.

\section{Implications for Cointegrating Regression}

To conclude, we return briefly to the implications of our results for asymptotic inference in regression models. Let

$$
p_{t}=\boldsymbol{q}_{t}^{\prime} \boldsymbol{\beta}+y_{t}
$$

where $\boldsymbol{q}_{t}=\sum_{s=1}^{t} \boldsymbol{x}_{s}$, and $\boldsymbol{x}_{s} \sim \mathrm{I}\left(d_{X}\right)(k \times 1)$ and $y_{t} \sim \mathrm{I}\left(d_{Y}\right)$ for $-\frac{1}{2}<d_{Y}<\frac{1}{2}<1+d_{X}$, implying what is commonly called fractional cointegration. Suppose that the obvious generalizations of our Assumptions 1 and 2 hold, such that $\boldsymbol{u}_{t}$ is now a $k$-vector and

$$
\mathrm{E}\left[\begin{array}{c}
\boldsymbol{u}_{t} \\
w_{t}
\end{array}\right]\left[\begin{array}{ll}
\boldsymbol{u}_{t}^{\prime} & w_{t}
\end{array}\right]=\left[\begin{array}{ll}
\boldsymbol{\Omega}_{u u} & \boldsymbol{\omega}_{u w} \\
\boldsymbol{\omega}_{u w}^{\prime} & \omega_{w w}
\end{array}\right] \quad(k+1) \times(k+1) .
$$


The least squares estimator may be written in error-of-estimate form as

$$
\hat{\boldsymbol{\beta}}-\boldsymbol{\beta}=\left(\sum_{t=1}^{n} \boldsymbol{q}_{t} \boldsymbol{q}_{t}^{\prime}\right)^{-1}\left(\sum_{t=1}^{n} \boldsymbol{q}_{t-1} y_{t}+\sum_{t=1}^{n} \boldsymbol{x}_{t} y_{t}\right) .
$$

Suppose for simplicity that $L_{X}(n) \rightarrow 1$ and $L_{Y}(n) \rightarrow 1 .{ }^{6}$ Letting $\boldsymbol{X}$ be the vector of fractional Brownian motions such that $n^{-1 / 2-d_{X}} \boldsymbol{q}_{[n r]} \stackrel{d}{\rightarrow} \boldsymbol{X}(r)$ by Theorem 5.1, the continuous mapping theorem yields

$$
\frac{1}{n^{2+2 d_{X}}} \sum_{t=1}^{n} \boldsymbol{q}_{t} \boldsymbol{q}_{t}^{\prime} \stackrel{d}{\rightarrow} \int_{0}^{1} \boldsymbol{X} \boldsymbol{X}^{\prime} d r .
$$

Let $\boldsymbol{\Xi}_{X Y}, \boldsymbol{\Lambda}_{X Y}$ and $\boldsymbol{\Lambda}_{X Y}^{*}$ be $k \times 1$ vectors whose elements are the limits as defined in Proposition 5.1 with respect to each regressor, and let $\boldsymbol{\Sigma}_{X Y}=\operatorname{plim} n^{-1} \sum_{t=1}^{n} \boldsymbol{x}_{t} y_{t}(k \times 1)$ similarly. In the case where $\boldsymbol{\omega}_{u w} \neq \mathbf{0},{ }^{7}$ applying Proposition 5.1 shows that

$$
n^{\min \left\{1+d_{X}-d_{Y}, 1+2 d_{X}\right\}}(\hat{\boldsymbol{\beta}}-\boldsymbol{\beta}) \stackrel{d}{\rightarrow}\left(\int_{0}^{1} \boldsymbol{X} \boldsymbol{X}^{\prime} d r\right)^{-1} \boldsymbol{\Upsilon}_{X Y}
$$

where

$$
\boldsymbol{\Upsilon}_{X Y}=\left\{\begin{array}{cc}
\boldsymbol{\Xi}_{X Y}+\boldsymbol{\Lambda}_{X Y}, & d_{X}+d_{Y}>0 \\
\boldsymbol{\Xi}_{X Y}+\boldsymbol{\Lambda}_{X Y}^{*}+\boldsymbol{\Sigma}_{X Y}, & d_{X}+d_{Y}=0 \\
\boldsymbol{\Lambda}_{X Y}^{*}+\boldsymbol{\Sigma}_{X Y}, & d_{X}+d_{Y}<0
\end{array}\right.
$$

In the case $d_{X}+d_{Y}<0$, note how the endogeneity bias term dominates by an order of magnitude. The estimator becomes inconsistent as $d_{X}$ approaches $-\frac{1}{2}$, which is the stationarity boundary for $\boldsymbol{q}_{t}$. This generalizes the well-known fact that cointegrating regressions are consistent with endogenous regressors, whereas stationary regressions are not, and locates the borderline precisely.

On the other hand, if $\boldsymbol{\omega}_{u w}=0$ and hence $\boldsymbol{q}_{t}$ is strictly exogenous, we obtain

$$
n^{1+d_{X}-d_{Y}}(\hat{\boldsymbol{\beta}}-\boldsymbol{\beta}) \stackrel{d}{\rightarrow}\left(\int_{0}^{1} \boldsymbol{X} \boldsymbol{X}^{\prime} d r\right)^{-1} \boldsymbol{\Xi}_{X Y}
$$

for all $d_{X}$ and $d_{Y}$ in the assumed range. In this case the estimator is consistent except where $d_{X}$ is near to $d_{Y}-1$, a region which may include stationary processes.

However, except when $d_{Y}=0$, note that the structure of $\boldsymbol{\Xi}_{X Y}$ appears to preclude mixed normal inference. Specifically, while both $\boldsymbol{\Xi}_{1, X Y}$ and $\boldsymbol{\Xi}_{3, X Y}$ appear conditionally Gaussian in the usual way, the conditioning processes are different. There exist no simple operations involving linear data projection by which the variance of $\boldsymbol{\Xi}_{X Y} \mid \boldsymbol{X}$ may be computed, and hence no prospect of implementing efficient mixed Gaussian estimators of the type developed by Hansen and Phillips (1990) and Saikkonen (1991). Further note, in connection with tests of the Johansen (1991) type, that autoregressive filters cannot reduce fractional processes to white noise. Our findings therefore have important implications for the interpretation of standard methods when the shortrun autocorrelation structure of cointegration models is unknown, and could involve long memory.

\footnotetext{
${ }^{6}$ There is no loss of generality in these limits since $\boldsymbol{\Omega}$ is unrestricted; compare the discussion following Proposition 2.1 .

${ }^{7}$ Assume for simplicity that all the elements are nonzero, otherwise convergence rates can differ.
} 


\section{A Appendix: Proofs}

\section{A.1 Proof of Proposition 2.1}

The coefficient of $L^{j}$ in the expansion of $b(L)=\theta(L) a(L)$ is

$$
b_{j}=\sum_{i=0}^{j} \theta_{i} a_{j-i} \sim \frac{1}{\Gamma\left(d_{X}\right)} \sum_{i=0}^{j-1} \theta_{i}(j-i)^{d_{X}-1} .
$$

Therefore, for any $\eta>1$ note that

$$
b_{j} \sim \frac{j^{d_{X}-1}}{\Gamma\left(d_{X}\right)}\left(\frac{j-j^{1 / \eta}}{j}\right)^{d_{X}-1} \sum_{i=0}^{j-1} \theta_{i}\left(\frac{j-i}{j-j^{1 / \eta}}\right)^{d_{X}-1} .
$$

Write

$$
\sum_{i=0}^{j-1} \theta_{i}\left(\frac{j-i}{j-j^{1 / \eta}}\right)^{d_{X}-1}=A(j)+B(j)
$$

where

$$
A(j)=\sum_{i=0}^{\left[j^{1 / \eta}\right]-1} \theta_{i}\left(\frac{j-i}{j-j^{1 / \eta}}\right)^{d_{X}-1}
$$

and

$$
B(j)=\sum_{i=\left[j^{1 / \eta}\right]}^{j-1} \theta_{i}\left(\frac{j-i}{j-j^{1 / \eta}}\right)^{d_{X}-1} .
$$

Since the $\theta_{i}$ are summable and $(j-i) /\left(j-j^{1 / \eta}\right) \rightarrow 1$ as $j \rightarrow \infty$ for each fixed $i \geq 0, A(j) \rightarrow \theta(1)$ as $j \rightarrow \infty$. To show that $B(j) \rightarrow 0$, define $k=j-i$. Since $\theta_{i}=O\left(i^{-1-\delta}\right)$ for $\delta>0$ by assumption,

$$
\begin{aligned}
B(j) & \leq \sum_{i=\left[j^{1 / \eta}\right]}^{j-1}\left|\theta_{i}\right|\left(\frac{j-i}{j-j^{1 / \eta}}\right)^{d_{X}-1} \\
& =O\left(\left(j-j^{1 / \eta}\right)^{1-d_{X}} j^{-(1+\delta) / \eta} \sum_{k=1}^{j-\left[j^{1 / \eta}\right]}\left(\frac{j-k}{j^{1 / \eta}}\right)^{-1-\delta} k^{d_{X}-1}\right) \\
& =O\left(\left(j-j^{1 / \eta}\right) j^{-(1+\delta) / \eta}\right)
\end{aligned}
$$

in view of the fact that $j-k \geq j^{1 / \eta}$ for all $k$. Since $\eta>1$ is arbitrary, pick $\eta<1+\delta$ to complete the proof.

\section{A.2 Proof of Proposition 3.1}

Under Assumption 1,

$$
\begin{aligned}
\mathrm{E}\left(G_{2 n}\right) & =\frac{1}{K(n)} \sum_{k=0}^{\infty} b_{k} \sum_{j=k+1}^{k+n-1} c_{j} \sum_{i=1-k}^{n-j} \mathrm{E}\left(u_{i} w_{i}\right) \\
& =\frac{\omega_{u w}}{K(n)} \sum_{k=0}^{\infty} b_{k} \sum_{t=1}^{n-1}(n-t) c_{k+t} .
\end{aligned}
$$


where the second equality follows upon the substitution $t=j-k$. It can be verified that

$$
\begin{aligned}
\sum_{k=0}^{\infty} b_{k} \sum_{t=1}^{n-1}(n-t) c_{k+t} & =\sum_{t=1}^{n-1} \sum_{s=0}^{t-1}\left(\sum_{k=0}^{s} b_{k}\right) c_{s+1}+\sum_{t=1}^{n-1} \sum_{s=t}^{\infty}\left(\sum_{k=s-t+1}^{s} b_{k}\right) c_{s+1} \\
& =\sum_{t=1}^{n-1} \sum_{s=0}^{t-1} a_{n, t-s}(t / n, 0) c_{s+1}+\sum_{t=1}^{n-1} \sum_{s=t}^{\infty} a_{n, t-s}(t / n, 0) c_{s+1}
\end{aligned}
$$

where the expression

$$
a_{n t}\left(s, s^{\prime}\right)=\sum_{j=\max \left\{0,\left[n s^{\prime}\right]-t+1\right\}}^{[n s]-t} b_{j}
$$

is defined in DDJ, equation (3.2). According to a straightforward extension of DDJ Lemma 3.1,

$$
a_{n,[n s]-[n x]}(s, 0) \sim\left\{\begin{array}{cc}
\frac{L_{X}(n)[n x]^{d_{X}}}{\Gamma\left(d_{X}+1\right)}, & 0 \leq x \leq s \\
L_{X}(n) \frac{[n x]^{d_{X}}-([n x]-[n s])^{d_{X}}}{\Gamma\left(d_{X}+1\right)}, & x>s .
\end{array}\right.
$$

In the case $d_{X}+d_{Y}>0$, applying Assumption 2 and substituting $d_{Y} / \Gamma\left(d_{Y}+1\right)$ for $1 / \Gamma\left(d_{Y}\right)$, we have

$$
\begin{aligned}
\frac{1}{K(n)} \sum_{t=1}^{n-1} \sum_{s=0}^{t-1} a_{n, t-s}(t / n, 0) c_{s+1} & \sim \frac{d_{Y}}{n^{2} \Gamma\left(d_{X}+1\right) \Gamma\left(d_{Y}+1\right)} \sum_{t=1}^{n-1} \sum_{s=1}^{t}\left(\frac{s}{n}\right)^{d_{X}+d_{Y}-1} \\
& \rightarrow \frac{d_{Y}}{\Gamma\left(d_{X}+1\right) \Gamma\left(d_{Y}+1\right)} \int_{0}^{1} \int_{0}^{\tau} \zeta^{d_{X}+d_{Y}-1} d \zeta d \tau \\
& =\frac{d_{Y}}{\Gamma\left(d_{X}+1\right) \Gamma\left(d_{Y}+1\right)\left(d_{Y}+d_{X}\right)\left(1+d_{Y}+d_{X}\right)}
\end{aligned}
$$

Similarly,

$$
\begin{aligned}
& \frac{1}{K(n)} \sum_{t=1}^{n-1} \sum_{s=t}^{\infty} a_{n, t-s}(t / n, 0) c_{s+1} \\
& \sim \frac{d_{Y}}{n^{2} \Gamma\left(d_{X}+1\right) \Gamma\left(d_{Y}+1\right)} \sum_{t=1}^{n-1} \sum_{s=0}^{\infty}\left(\left(\frac{s+t}{n}\right)^{d_{X}}-\left(\frac{s}{n}\right)^{d_{X}}\right)\left(\frac{s+t}{n}\right)^{d_{Y}-1} \\
& \rightarrow \frac{d_{Y}}{\Gamma\left(d_{X}+1\right) \Gamma\left(d_{Y}+1\right)} \int_{0}^{\infty} \int_{0}^{1}\left((\zeta+\tau)^{d_{X}}-\tau^{d_{X}}\right)(\zeta+\tau)^{d_{Y}-1} d \zeta d \tau \\
& =\frac{1}{\Gamma\left(d_{X}+1\right) \Gamma\left(d_{Y}+1\right)\left(d_{X}+d_{Y}\right)} \\
& \quad \times \int_{0}^{\infty}\left[d_{Y}(1+\tau)^{d_{X}+d_{Y}}+d_{X} \tau^{d_{X}+d_{Y}}-\left(d_{X}+d_{Y}\right)(1+\tau)^{d_{Y}} \tau^{d_{X}}\right] d \tau .
\end{aligned}
$$

Combining these two limits completes the first part of the proof for the cases with $d_{Y} \neq 0$. If $d_{Y}=0$, Assumption 2(b) does not permit the explicit representation used in (A-6) and (A-7). However, summability of the $c_{s}$ coefficients implies that

$$
\sum_{t=1}^{n-1} \sum_{s=0}^{\infty} a_{n, t-s}(t / n, 0) c_{s+1}=o\left(n^{1+d_{X}} L_{X}(n)\right)
$$

and $\mathrm{E}\left(G_{2 n}\right)$ vanishes in the limit. These expressions are therefore formally correct in all the cases. 


\section{A.3 Proof of Proposition 3.2}

Let $\mathcal{L}_{X Y}$ denote the integral in (3.4), and let $f_{X Y}(\tau)$ denote the integrand such that $\mathcal{L}_{X Y}=$ $\int_{0}^{\infty} f_{X Y}(\tau) d \tau$. For $0<d_{X}, d_{Y}<1 / 2, \lim _{\tau \rightarrow \pm \infty} f_{X Y}(\tau)=0, \lim _{\tau \rightarrow 0} f_{X Y}(\tau)=d_{Y}$, and the function is integrable for all $\tau$. For $-\frac{1}{2}<d_{X}<0$ we have $\lim _{\tau \rightarrow \pm \infty} f_{X Y}(\tau)=0$, and $f_{X Y}(\tau)$ has a singularity at $\tau=0$ with $\lim _{\tau \rightarrow 0} f_{X Y}(\tau) \tau^{-\left(d_{X}+d_{Y}\right)}=d_{X}$, and $f_{X Y}(\tau)$ is integrable. For $-\frac{1}{2}<d_{Y}<0$ it has a singularity at $\tau=-1$ with $\lim _{\tau \rightarrow-1} f_{X Y}(\tau)(\tau+1)^{-\left(d_{X}+d_{Y}\right)}=d_{Y}$, and is again integrable.

Consider an auxiliary integral $\mathcal{L}_{X Y}^{*}=\int_{-\infty}^{\infty} f_{X Y}(\tau) d \tau$. Changing the variable of integration, $\tau+1=-t$, observe that

$$
\mathcal{L}_{X Y}^{*}=(-1)^{d_{X}+d_{Y}} \mathcal{L}_{Y X}^{*}=(-1)^{2\left(d_{X}+d_{Y}\right)} \mathcal{L}_{X Y}^{*}
$$

and hence $\mathcal{L}_{X Y}^{*}=\mathcal{L}_{Y X}^{*}=0$. Divide the range of integration in $\mathcal{L}_{Y X}^{*}$ into intervals $(-\infty,-1)$, $(-1,0)$, and $(0, \infty)$. We can then show $\int_{-\infty}^{-1} f_{Y X}(t) d t=(-1)^{d_{X}+d_{Y}} \mathcal{L}_{X Y}$ and also

$$
\int_{-1}^{0} f_{Y X}(t) d t=\frac{(-1)^{d_{X}+d_{Y}} d_{Y}+d_{X}}{d_{X}+d_{Y}+1}-(-1)^{d_{Y}}\left(d_{X}+d_{Y}\right) B\left(d_{X}+1, d_{Y}+1\right),
$$

whereas the integral over the third interval is simply $\mathcal{L}_{Y X}$. Adding the three integrals we obtain

$$
(-1)^{d_{X}+d_{Y}} \mathcal{L}_{X Y}+\frac{(-1)^{d_{X}+d_{Y}} d_{Y}+d_{X}}{d_{X}+d_{Y}+1}-(-1)^{d_{Y}}\left(d_{X}+d_{Y}\right) B\left(d_{X}+1, d_{Y}+1\right)+\mathcal{L}_{Y X}=0
$$

and by symmetry, noting $B(x, y)=B(y, x)$,

$$
(-1)^{d_{X}+d_{Y}} \mathcal{L}_{Y X}+\frac{(-1)^{d_{X}+d_{Y}} d_{X}+d_{Y}}{d_{X}+d_{Y}+1}-(-1)^{d_{X}}\left(d_{X}+d_{Y}\right) B\left(d_{X}+1, d_{Y}+1\right)+\mathcal{L}_{X Y}=0
$$

Now multiply (A-9) by $(-1)^{d_{X}+d_{Y}}$, subtract from (A-10), and rearrange to get.

$$
\mathcal{L}_{X Y}=-\frac{d_{Y}}{d_{X}+d_{Y}+1}+(-1)^{d_{X}} \frac{1-(-1)^{2 d_{Y}}}{1-(-1)^{2\left(d_{X}+d_{Y}\right)}}\left(d_{X}+d_{Y}\right) B\left(d_{X}+1, d_{Y}+1\right) .
$$

Using $(-1)^{x}=e^{i \pi x}$ and standard trigonometric identities,

$$
(-1)^{d_{X}} \frac{1-(-1)^{2 d_{Y}}}{1-(-1)^{2\left(d_{X}+d_{Y}\right)}}=\frac{\sin \pi d_{Y}}{\sin \pi\left(d_{X}+d_{Y}\right)}
$$

and therefore

$$
\mathcal{L}_{X Y}=-\frac{d_{Y}}{d_{X}+d_{Y}+1}+\left(d_{X}+d_{Y}\right) B\left(d_{X}+1, d_{Y}+1\right) \frac{\sin \pi d_{Y}}{\sin \pi\left(d_{X}+d_{Y}\right)} .
$$

The proof is completed by substituting this expression into (3.4) and rearranging, using the identities $B(x, y)=\Gamma(x) \Gamma(y) / \Gamma(x+y), \Gamma(1-x) \Gamma(x)=\pi / \sin \pi x$ and $\Gamma(x+1)=x \Gamma(x)$.

\section{A.4 Proof of Proposition 3.3}

In this case, note that if $a_{n t}$ is defined by (A-5) then

$$
a_{n, t-s}(t / n, 0) c_{s+1}=O\left(s^{d_{X}+d_{Y}-1} L_{X}(s) L_{Y}(s)\right)
$$


so that these terms are summable by assumption. Considering expression (A-4), the proposition follows since

$$
\sum_{t=1}^{n-1} \sum_{s=0}^{t-1} a_{n, t-s}(t / n, 0) c_{s+1}=O(n)
$$

and

$$
\sum_{t=1}^{n-1} \sum_{s=t}^{\infty} a_{n, t-s}(t / n, 0) c_{s+1}=o(n)
$$

\section{A.5 Proof of Theorem 3.1}

Setting $i=s-k$, rewrite $(3.2)$ as

$$
\begin{aligned}
G_{2 n}-\mathrm{E}\left(G_{2 n}\right) & =\frac{1}{K(n)} \sum_{t=1}^{n-1} \sum_{s=1}^{t} \sum_{k=0}^{\infty} b_{k} c_{k+t-s+1}\left(u_{s-k} w_{s-k}-\omega_{u w}\right) \\
& =\frac{1}{K(n)} \sum_{t=1}^{n-1} \sum_{s=1}^{t} P_{t s}
\end{aligned}
$$

where

$$
P_{t s} \equiv \sum_{i=-\infty}^{s} b_{s-i} c_{t+1-i}\left(u_{i} w_{i}-\omega_{u w}\right)
$$

Hence note that

$$
\mathrm{E}\left(G_{2 n}-\mathrm{E}\left(G_{2 n}\right)\right)^{2} \leq \frac{2}{K(n)^{2}} \sum_{t=1}^{n-1} \sum_{s=1}^{t} \sum_{m=0}^{t-s} \sum_{k=0}^{s-1} \mathrm{E}\left(P_{t s} P_{t-m, s-k}\right)
$$

where, letting $C$ denote a generic finite constant, and defining $j=s-i$,

$$
\begin{aligned}
\mathrm{E}\left(P_{t s} P_{t-m, s-k}\right) & =\frac{\mu_{u w}^{4}-\omega_{u w}^{2}}{K(n)^{2}} \sum_{i=-\infty}^{s-k} b_{s-i} b_{s-k-i} c_{t+1-i} c_{t-m+1-i} \\
& =\frac{\mu_{u w}^{4}-\omega_{u w}^{2}}{K(n)^{2}} \sum_{j=k}^{\infty} b_{j} b_{j-k} c_{t+1-s+j} c_{t-m+1-s+j} \\
& \leq \frac{C}{n^{2\left(1+d_{X}+d_{Y}\right)}} \sum_{j=k}^{\infty} j^{d_{X}-1}(j-k)^{d_{X}-1}(j+t+1-s)^{d_{Y}-1}(j+t-m+1-s)^{d_{Y}-1} \\
& \leq \frac{C}{n^{2\left(1+d_{X}+d_{Y}\right)}} k^{2 d_{X}-1}(k+t+1-s)^{d_{Y}-1}(k+t-m+1-s)^{d_{Y}-1} .
\end{aligned}
$$

Hence,

$$
\mathrm{E}\left(G_{2 n}-\mathrm{E}\left(G_{2 n}\right)\right)^{2} \leq \frac{C}{n^{2\left(1+d_{X}+d_{Y}\right)}} \sum_{t=1}^{n-1} \sum_{s=1}^{t}(s-1)^{2 d_{X}}(t+1-s)^{d_{Y}-1} \sum_{m=0}^{t-s}(t-m+1-s)^{d_{Y}-1} .
$$

By conventional summation arguments (Davidson 1994, Thm 2.27) and also applying Lemma A.1 of DDJ in the case $d_{X}<0$, these sums can be bounded as follows.

Case $d_{Y}>0$ :

$$
\mathrm{E}\left(G_{2 n}-\mathrm{E}\left(G_{2 n}\right)\right)^{2} \leq \frac{C}{n^{2\left(1+d_{X}+d_{Y}\right)}} \sum_{t=1}^{n-1} \sum_{s=1}^{t}(t+1-s)^{2 d_{Y}-1}(s-1)^{2 d_{X}}
$$




$$
=O\left(n^{-1}\right) \text {. }
$$

Case $d_{Y} \leq 0$ :

$$
\begin{aligned}
\mathrm{E}\left(G_{2 n}-\mathrm{E}\left(G_{2 n}\right)\right)^{2} & \leq \frac{C}{n^{2\left(1+d_{X}+d_{Y}\right)}} \sum_{t=1}^{n-1} \sum_{s=1}^{t}(t+1-s)^{d_{Y}-1}(s-1)^{2 d_{X}} \\
& =\left\{\begin{array}{cc}
O\left(n^{-1} \log n\right), & d_{Y}=0 \\
O\left(n^{-1-2 d_{Y}}\right), & d_{Y}<0
\end{array} .\right.
\end{aligned}
$$

\section{A.6 Proof of Theorem 3.2}

First note that

$$
\begin{aligned}
\mathrm{E}\left(\frac{1}{n} \sum_{t=1}^{n} x_{t} y_{t}-\sigma_{X Y}\right)^{2} & =\frac{1}{n^{2}} \sum_{k=0}^{\infty} \sum_{j=0}^{\infty} \sum_{i=0}^{\infty} \sum_{l=0}^{\infty} b_{k} b_{l} c_{j} c_{i} \\
& \times \sum_{t=1}^{n-1} \sum_{s=1}^{n-1}\left[\mathrm{E}\left(u_{t-k} w_{t-j} u_{s-l} w_{s-i}\right)-\mathrm{E}\left(u_{t-k} w_{t-j}\right) \mathrm{E}\left(u_{s-l} w_{s-i}\right)\right] .
\end{aligned}
$$

Under the assumptions,

$$
\begin{aligned}
\mathrm{E}\left(u_{t-k} w_{t-j} u_{s-l} w_{s-i}\right)-\mathrm{E}\left(u_{t-k} w_{t-j}\right) \mathrm{E}\left(u_{s-l} w_{s-i}\right) \\
=\left\{\begin{array}{cc}
\mu_{u w}^{4}-\omega_{u w}^{2} & t-k=s-l=t-j=s-i \\
\omega_{u u} \omega_{w w}-\omega_{u w}^{2} & t-k=s-l \neq t-j=s-i \\
0 & \text { otherwise. }
\end{array}\right.
\end{aligned}
$$

Collecting these terms and letting sums over an empty index set equal zero yields

$$
\begin{aligned}
\mathrm{E}\left(\frac{1}{n} \sum_{t=1}^{n} x_{t} y_{t}-\sigma_{X Y}\right)^{2} & =\frac{\mu_{u w}^{4}-\omega_{u w}^{2}}{n} \sum_{k=0}^{\infty} b_{k}^{2} c_{k}^{2}+\frac{\omega_{u u} \omega_{w w}-\omega_{u w}^{2}}{n} \sum_{k=0}^{\infty} b_{k}^{2} \sum_{j=0}^{\infty} c_{j}^{2} \\
& =O\left(n^{-1}\right) .
\end{aligned}
$$

The conditions of part (ii) overlap with those of part (i), but ensure that the sequences $\left\{b_{k}\right\}$ and $\left\{c_{j}\right\}$ are absolutely summable. In this case,

$$
\begin{aligned}
n^{-1 / 2} \sum_{t=1}^{n} x_{t} y_{t} & =\sum_{j=0}^{\infty} \sum_{k=0}^{\infty} b_{k} c_{j}\left(n^{-1 / 2} \sum_{t=1}^{n} u_{t-k} w_{t-j}\right) . \\
& \stackrel{d}{\rightarrow} \sum_{j=0}^{\infty} \sum_{k=0}^{\infty} b_{k} c_{j} Z(j, k)=\zeta
\end{aligned}
$$

(say) where, since the terms $u_{t-k} w_{t-j}$ are i.i.d. with mean 0 and finite variance $\omega_{u u} \omega_{w w}$ for each pair $(j, k)$, the $Z(j, k)$ are $N\left(0, \omega_{u u} \omega_{w w}\right)$ random variables, by the standard Lindeberg-Lévy CLT. Note that $Z(j, k)=Z\left(j^{\prime}, k^{\prime}\right)$ if $j-k=j^{\prime}-k^{\prime}$ and $\mathrm{E}\left(Z(j, k) Z\left(j^{\prime}, k^{\prime}\right)\right)=0$ if $j-k \neq j^{\prime}-k^{\prime}$. Hence $\zeta$ is normally distributed with zero mean and variance

$$
\begin{aligned}
V & =\omega_{u u} \omega_{w w}\left[\sum_{k=0}^{\infty} b_{k}^{2} \sum_{j=0}^{\infty} c_{j}^{2}+2 \sum_{p=1}^{\infty}\left(\sum_{k=0}^{\infty} b_{k} b_{k+p} \sum_{j=0}^{\infty} c_{j} c_{j+p}\right)\right] \\
& <\infty
\end{aligned}
$$




\section{A.7 Proof of Lemma 4.1}

A preliminary lemma is needed as follows.

Lemma A.1 For $0<\alpha<\frac{1}{2}$ and $a>b \geq 0$, and $L_{X}(n)$ slowly varying,

$$
\left|\frac{1}{n} \sum_{s=1+[n b]}^{[n a]}\left(\frac{s}{n}\right)^{\alpha-1} \frac{L_{X}(s)}{L_{X}(n)}-\int_{b}^{a} u^{\alpha-1} d u\right|=o(1) .
$$

Proof This follows since

$$
\frac{1}{n} \sum_{s=1+[n b]}^{[n a]}\left(\frac{s}{n}\right)^{\alpha-1} \frac{L_{X}(s)}{L_{X}(n)}=\frac{1}{n} \sum_{s=1+[n b]}^{[n a]}\left(\frac{s}{n}\right)^{\alpha-1}+o(1)
$$

and

$$
\begin{aligned}
\frac{1}{n}\left|\sum_{s=1+[n b]}^{[n a]}\left[\left(\frac{s}{n}\right)^{\alpha-1}-n \int_{s / n}^{(s+1) / n} u^{\alpha-1} d u\right]\right| & =\frac{1}{n}\left|\sum_{s=1+[n b]}^{[n a]}\left[\left(\frac{s}{n}\right)^{\alpha-1}-\frac{n}{\alpha}\left(\left(\frac{s+1}{n}\right)^{\alpha}-\left(\frac{s}{n}\right)^{\alpha}\right)\right]\right| \\
& \leq \frac{1}{n}\left|\sum_{s=1+[n b]}^{[n a]}\left[\left(\frac{s+1}{n}\right)^{\alpha-1}-\left(\frac{s}{n}\right)^{\alpha-1}\right]\right| \\
& =\frac{1}{n}\left|\left(\frac{[n a]+1}{n}\right)^{\alpha-1}-\left(\frac{[n b]+1}{n}\right)^{\alpha-1}\right|=O\left(n^{-1}\right) .
\end{aligned}
$$

Considering the components of equation (4.2), define $q$ and $u$ by $t-m=[n q]$ and $s-i=[n u]$. If $d_{X}>0$, write

$$
\frac{b_{[n u]}}{n^{d_{X}} L_{X}(n)}=\frac{(([n u]+1) / n)^{d_{X}-1}}{n \Gamma\left(d_{X}\right)} \frac{L_{X}(n u)}{L_{X}(n)}
$$

Note by Lemma A.1 that in this case,

$$
\begin{aligned}
\frac{1}{n^{d_{X}} L_{X}(n)} \sum_{s=\max \{0,1-[n p]\}}^{[n q]+[n r]-[n p]} b_{s} & =\frac{1}{\Gamma\left(d_{X}\right)} \int_{\max \{0,-p\}}^{q+r-p} u^{d_{X}-1} d u+o(1) \\
& =\frac{(q+r-p)^{d_{X}}-1_{\{p<0\}}(-p)^{d_{X}}}{\Gamma\left(1+d_{X}\right)}+o(1) .
\end{aligned}
$$

If $d_{X}<0$, use part (c) of Assumption 2 to write

$$
\frac{b_{[n u]}}{n^{d_{X}} L_{X}(n)}=\frac{(([n u]+1) / n)^{d_{X}}-([n u] / n)^{d_{X}}}{\Gamma\left(1+d_{X}\right)}+o(1)
$$

for $[n u]>0$ where $b_{0}=L_{X}(0)$, and hence, as before,

$$
\frac{1}{n^{d_{X}} L_{X}(n)} \sum_{s=\max \{0,1-[n p]\}}^{[n q]+[n r]-[n p]} b_{s}=\frac{(q+r-p)^{d_{X}}-1_{\{p<0\}}(-p)^{d_{X}}}{\Gamma\left(1+d_{X}\right)}+o(1) .
$$

In the case $d_{X}=0$, under part (b) of Assumption 2, note that

$$
\sum_{s=\max \{0,1-[n p]\}}^{[n q]+[n r]-[n p]} b_{s}= \begin{cases}O(1) & p \geq 0 \\ o(1) & p<0\end{cases}
$$


which is formally equivalent to (A-13) when $L_{X}(n)$ is defined as a constant not depending on $n$. Moreover, the sum can be assigned the limiting value $1-1_{\{p<0\}}$ by choice of normalization, without loss of generality.

Proceeding similarly, for the case $d_{Y}>0$ we may now write

$$
\frac{c_{[n q]}}{n^{d_{Y}} L_{Y}(n)}=\frac{(([n q]+1) / n)^{d_{Y}-1}}{n \Gamma\left(d_{Y}\right)} \frac{L_{Y}(n q)}{L_{Y}(n)}
$$

and hence, from (4.2), $a_{n[n p][n r]}=A_{X Y}(r, p)+o(1)$ where

$$
\begin{aligned}
A_{X Y}(r, p)= & \frac{1}{\Gamma\left(1+d_{X}\right) \Gamma\left(d_{Y}\right)} \int_{\max \{0,-r\}}^{1-r} q^{d_{Y}-1}\left[(q+r-p)^{d_{X}}-1_{\{p<0\}}(-p)^{d_{X}}\right] d q \\
= & \frac{1}{\Gamma\left(1+d_{X}\right) \Gamma\left(d_{Y}\right)} \int_{\max \{0,-r\}}^{1-r} q^{d_{Y}-1}(q+r-p)^{d_{X}} d q \\
& -\frac{1_{\{p<0\}}(-p)^{d_{X}}\left[(1-r)^{d_{Y}}-1_{\{r<0\}}(-r)^{d_{Y}}\right]}{\Gamma\left(1+d_{X}\right) \Gamma\left(1+d_{Y}\right)}
\end{aligned}
$$

To verify that this formula matches (4.3) see Abramovitz and Stegun (1972), 15.3.1.

In the case $d_{Y}<0$, on the other hand,

$$
\begin{aligned}
\frac{c_{[n q]}}{n^{d_{Y}} L_{Y}(n)} & =\frac{(([n q]+1) / n)^{d_{Y}}-([n q]+1 / n)^{d_{Y}}}{\Gamma\left(d_{Y}\right)}+o(1) \\
& =\frac{(([n q]+\lambda) / n)^{d_{Y}-1}}{\Gamma\left(1+d_{Y}\right)}+o(1), \quad 0 \leq \lambda \leq 1 .
\end{aligned}
$$

The approximation in (4.3) may be applied as before in respect of the first and second terms. However, since the integral of the increments in (A-15) diverges at 0 , the terms with factor $1_{\{p<0\}}$ in (4.3) have to be constructed as the limiting case of

$$
\frac{1}{n^{d_{Y}} L_{Y}(n)} \sum_{t=\max \{1-[n r], 0\}}^{n-1-[n r]} c_{t}=\frac{((n-1-[n r]) / n)^{d_{Y}}-1_{\{r<0\}}(-[n r] / n)^{d_{Y}}}{\Gamma\left(1+d_{Y}\right)}+o(1) .
$$

The expression in (A-14) nonetheless continues to apply.

\section{A.8 Proof of Lemma 4.2}

By arguments closely paralleling those of Lemma 4.1 applied to the formula in (4.4), we arrive at

$$
\begin{aligned}
\mathrm{E}_{X Y}(p, r)= & \frac{1}{\Gamma\left(d_{Y}+1\right) \Gamma\left(d_{X}\right)} \int_{\max \{0,-p\}}^{1-p} u^{d_{X}-1}\left[(1-r)^{d_{Y}}-(u+p-r)^{d_{Y}}\right] d u \\
= & \frac{1}{\Gamma\left(d_{Y}+1\right) \Gamma\left(d_{X}+1\right)}\left[(1-r)^{d_{Y}}(1-p)^{d_{X}}-d_{X} \int_{0}^{1-p} u^{d_{X}-1}(u+p-r)^{d_{Y}} d u\right. \\
& \left.\quad 1_{\{p<0\}}\left((1-r)^{d_{Y}}(-p)^{d_{X}}-d_{X} \int_{0}^{-p} u^{d_{X}-1}(u+p-r)^{d_{Y}} d u\right)\right]
\end{aligned}
$$

This yields the stated result by routine manipulation and application of Abramowitz and Stegun (1972), 15.3.1. 


\section{A.9 Proof of Proposition 4.1}

For a function $F$ and fractional Brownian motion $X$ as defined in (1.2), define the notation $\int F \delta X$ by the formula

$$
\int F \delta X=\frac{1}{\Gamma\left(d_{X}+1\right)} \int_{-\infty}^{1} F(\tau)\left[(1-\tau)^{d_{X}}-1_{\{\tau<0\}}(-\tau)^{d_{X}}\right] d U(\tau)
$$

and define $\int F \delta Y$ similarly. The idea here is that $\int F \delta X$ represents a notion of 'integral with respect to fractional Brownian motion', and in particular, $\int \delta X=X(1)$ and $\int \delta Y=Y(1)$. However, we need to emphasize that these are not in the class of objects that is the subject of this paper, for which the notations $\int F d X$ and $\int F d Y$ are customary.

Observe that

$$
A_{X Y}(r, p)+E_{Y X}(r, p)=\frac{\left[(1-r)^{d_{Y}}-1_{\{r<0\}}(-r)^{d_{Y}}\right]\left[(1-p)^{d_{X}}-1_{\{p<0\}}(-p)^{d_{X}}\right]}{\Gamma\left(d_{Y}+1\right) \Gamma\left(d_{X}+1\right)}
$$

with the corresponding identity for $A_{Y X}(p, r)+E_{X Y}(p, r)$. Therefore, defining processes

$$
\begin{aligned}
& \tilde{X}(t)=\frac{1}{\Gamma\left(d_{X}+1\right)} \int_{-\infty}^{t}\left[(1-\tau)^{d_{X}}-1_{\{\tau<0\}}(-\tau)^{d_{X}}\right] d U(\tau) \\
& \tilde{Y}(\tau)=\frac{1}{\Gamma\left(d_{Y}+1\right)} \int_{-\infty}^{\tau}\left[(1-t)^{d_{X}}-1_{\{t<0\}}(-t)^{d_{X}}\right] d W(t)
\end{aligned}
$$

note that

$$
\Xi_{X Y}+\Xi_{Y X}=\int \tilde{X} \delta Y+\int \tilde{Y} \delta X
$$

Next define

$$
\breve{X}(t)= \begin{cases}\frac{1}{\Gamma\left(d_{X}+1\right)} \int_{t}^{1}(1-\tau)^{d_{X}} d U(\tau) & t \geq 0 \\
\frac{1}{\Gamma\left(d_{X}+1\right)}\left(\begin{array}{c}
\int_{0}^{1}(1-\tau)^{d_{X}} d U(\tau) \\
+\int_{t}^{0}\left[(1-\tau)^{d_{X}}-(-\tau)^{d_{X}}\right] d U(\tau)
\end{array}\right) & t<0 .\end{cases}
$$

and also the complementary expression for $\breve{Y}(\tau)$, with $d_{Y}$ replacing $d_{X}, W$ replacing $U$, and $t, \tau$ interchanged. It can easily be verified that $X(1)=\tilde{X}(t)+\breve{X}(t)$ for any $t<1$ and likewise $Y(1)=\tilde{Y}(\tau)+\breve{Y}(\tau)$ for any $\tau<1$. Therefore, given (A-17) and (A-16), we find

$$
2 X(1) Y(1)=\Xi_{X Y}+\Xi_{Y X}+\int \breve{X} \delta Y+\int \breve{Y} \delta X .
$$

Observe that, since $\mathrm{E}(X(1) Y(1))=\psi_{X Y}$ while $\mathrm{E}\left(\Xi_{X Y}\right)=\mathrm{E}\left(\Xi_{Y X}\right)=0$, (A-19) implies

$$
\mathrm{E}\left(\int \breve{X} \delta Y\right)+\mathrm{E}\left(\int \breve{Y} \delta X\right)=2 \psi_{X Y} .
$$

Next, combining (A-18) with (A-16) consider the following decomposition.

$$
\begin{aligned}
\int \breve{X} \delta Y= & \frac{1}{\Gamma\left(d_{Y}+1\right)}\left(\int_{0}^{1}(1-t)^{d_{Y}} \breve{X}(t) d W(t)+\int_{-\infty}^{0}\left[(1-t)^{d_{Y}}-(-t)^{d_{Y}}\right] \breve{X}(t) d W(t)\right) \\
= & \frac{1}{\Gamma\left(d_{X}+1\right) \Gamma\left(d_{Y}+1\right)} \int_{0}^{1}\left(\int_{t}^{1}(1-\tau)^{d_{X}} d U(\tau)\right)(1-t)^{d_{Y}} d W(t) \\
& +\frac{1}{\Gamma\left(d_{X}+1\right) \Gamma\left(d_{Y}+1\right)} \int_{-\infty}^{0}\left(\int_{0}^{1}(1-\tau)^{d_{X}} d U(\tau)\right)\left[(1-t)^{d_{Y}}-(-t)^{d_{Y}}\right] d W(t)
\end{aligned}
$$




$$
\begin{gathered}
+\frac{1}{\Gamma\left(d_{X}+1\right) \Gamma\left(d_{Y}+1\right)} \int_{-\infty}^{0}\left(\int_{t}^{0}\left[(1-\tau)^{d_{X}}-(-\tau)^{d_{X}}\right] d U(\tau)\right) \\
\times\left[(1-t)^{d_{Y}}-(-t)^{d_{Y}}\right] d W(t) \\
=\mathrm{E}\left(\int \breve{X} \delta Y\right)+\frac{1}{\Gamma\left(d_{X}+1\right) \Gamma\left(d_{Y}+1\right)} \int_{0}^{1}\left(\int_{0}^{\tau}(1-t)^{d_{Y}} d W(t)\right)(1-\tau)^{d_{X}} d U(\tau) \\
+\frac{1}{\Gamma\left(d_{X}+1\right) \Gamma\left(d_{Y}+1\right)}\left(\int_{-\infty}^{0}\left[(1-t)^{d_{Y}}-(-t)^{d_{Y}}\right] d W(t)\right)\left(\int_{0}^{1}(1-\tau)^{d_{X}} d U(\tau)\right) \\
+\frac{1}{\Gamma\left(d_{X}+1\right) \Gamma\left(d_{Y}+1\right)} \int_{-\infty}^{0}\left(\int_{-\infty}^{\tau}\left[(1-t)^{d_{Y}}-(-t)^{d_{Y}}\right] d W(t)\right) \\
\times\left[(1-\tau)^{d_{X}}-(-\tau)^{d_{X}}\right] d U(\tau) .
\end{gathered}
$$

In (A-21), note how the first and third terms involve non-adapted integrands, and hence must have non-zero means. However, these two-dimensional integrals can be formally rearranged as Itô integrals with respect to $d U(\tau)$ of $\mathcal{F}(\tau)$-measurable processes, provided the non-zero component means (corresponding to the diagonal contributions $t=\tau$ ) are included explicitly. This is done in the fourth member, labelled (A-22), noting that the two component means sum to $\mathrm{E}\left(\int \breve{X} \delta Y\right)$, which accordingly must appear explicitly. By direct calculation we find

$$
\begin{aligned}
\mathrm{E}\left(\int \breve{X} \delta Y\right)= & \frac{\omega_{u w}}{\Gamma\left(d_{X}+1\right) \Gamma\left(d_{Y}+1\right)} \times \\
& \left(\int_{0}^{1}(1-t)^{d_{X}+d_{Y}} d t+\int_{-\infty}^{0}\left[(1-t)^{d_{X}}-(-t)^{d_{X}}\right]\left[(1-t)^{d_{Y}}-(-t)^{d_{Y}}\right] d t\right) \\
= & \psi_{X Y}
\end{aligned}
$$

where the second equality is by (3.5). The remaining term in these expressions is the product of factors defined on $(-\infty, 0]$ and $(0, \xi]$ respectively, and hence has zero mean.

The corresponding decomposition can of course be carried out for $\int \breve{Y} \delta X$ whose mean, given the symmetry in $X$ and $Y$, is also $\psi_{X Y}$. Accordingly, consider the expression obtained by taking (A-21) and interchanging the pairs $(X, Y),(U, W)$, and $(t, \tau)$ throughout. The sum of this expression and (A-22) itself, after piecing together the integrals and simplifying, yields

$$
\int \breve{X} \delta Y+\int \breve{Y} \delta X=X(1) Y(1)+\psi_{X Y} .
$$

Equation (A-23) in combination with (A-19) yields the required formula.

\section{A.10 Proof of Lemmas 5.1 and 5.4}

The following preliminary lemmas are needed.

Lemma A.2 $\left|A_{X Y}(r, p)\right| \leq \bar{A}_{X Y}(r, p)$ where $A_{X Y}(r, p)$ is defined in (4.3) and

$$
\bar{A}_{X Y}(r, p)=\frac{\left|(1-r)^{d_{Y}}-1_{\{r<0\}}(-r)^{d_{Y}}\right|\left|(g-p)^{d_{X}}-1_{\{p<0\}}(-p)^{d_{X}}\right|}{\Gamma\left(d_{X}+1\right) \Gamma\left(d_{Y}+1\right)}
$$

where

$$
g= \begin{cases}1, & d_{X} \geq 0 \text { or } d_{X}<0, p<0 \\ r, & d_{X}<0, \quad p \geq 0\end{cases}
$$


Proof From (4.2),

$$
\begin{aligned}
\left|a_{n[n p][n r]}\right| & =\frac{n}{K(n)}\left|\sum_{t=\max \{1-[n r], 0\}}^{n-1-[n r]} c_{t}\left(\sum_{s=\max \{1-[n p], 0\}}^{t+[n r]-[n p]} b_{s}\right)\right| \\
& \leq \frac{n}{K(n)}\left|\sum_{t=\max \{1-[n r], 0\}}^{n-1-[n r]} c_{t}\right| \max _{\max \{1-[n r], 0\} \leq \tau \leq n-1}\left|\sum_{s=\max \{1-[n p], 0\}}^{\tau+[n r]-[n p]} b_{s}\right| \\
& =\frac{\left|(1-r)^{d_{Y}}-1_{\{r<0\}}(-r)^{d_{Y}} \max _{\max \{0, r\} \leq g \leq 1}\right|(g-p)^{d_{X}}-1_{\{p<0\}}(-p)^{d_{X}} \mid}{\Gamma\left(d_{X}+1\right) \Gamma\left(d_{Y}+1\right)}+o(1)
\end{aligned}
$$

as $n \rightarrow \infty$, using the same arguments as in the proof of Lemma 4.1. When $d_{X} \geq 0,(g-p)^{d_{X}}$ is monotone nondecreasing in $g$, and is maximized over [0,1] at $g=1$. When $d_{X}<0,(g-p)^{d_{X}}$ is monotone decreasing. If $p \geq 0$, so that $r \geq 0$, the maximum in (A-24) is achieved at $g=r$. On the other hand, if $p<0$ then $\left|(g-p)^{d_{X}}-1_{\{p<0\}}(-p)^{d_{X}}\right|$ is maximized at $g=1$, as indicated.

Lemma A.3 $\left|E_{X Y}(p, r)\right| \leq \bar{E}_{X Y}(p, r)$ where $E_{X Y}(p, r)$ is defined in (4.5) and

$$
\bar{E}_{X Y}(p, r)=\frac{\left|(1-p)^{d_{X}}-1_{\{p<0\}}(-p)^{d_{X}}\right|\left|(1-r)^{d_{Y}}-1_{\{r<0\}}(-r)^{d_{Y}}\right|}{\Gamma\left(d_{X}+1\right) \Gamma\left(d_{Y}+1\right)}
$$

Proof From (4.4)

$$
\begin{aligned}
\left|e_{n[n r][n p]}\right| & =\frac{n}{K(n)}\left|\sum_{s=\max \{0,1-[n p]\}}^{n-1-[n p]} b_{s}\left(\sum_{t=s+[n p]+1-[n r]}^{n-[n r]} c_{t}\right)\right| \\
& \leq \frac{n}{K(n)}\left|\sum_{s=\max \{0,1-[n p]\}}^{n-1-[n p]} b_{s}\right| \max _{\max \{0,1-[n p]\} \leq z \leq n-1}\left|\sum_{t=z+[n p]+1-[n r]}^{n-[n r]} c_{t}\right| \\
& =\frac{\left|(1-p)^{d_{X}}-1_{\{p<0\}}(-p)^{d_{X}}\right| \max _{\max \{0, p\} \leq g \leq 1}\left|(1-r)^{d_{Y}}-(g-r)^{d_{Y}}\right|}{\Gamma\left(d_{X}+1\right) \Gamma\left(d_{Y}+1\right)}+o(1)
\end{aligned}
$$

as $n \rightarrow \infty$. First, suppose $p \geq 0$. When $d_{Y} \geq 0$ then $(1-r)^{d_{Y}}-(g-r)^{d_{Y}}>0$ and is maximized over $[p, 1]$ at $g=p$, noting that $r \leq p$ in this case. When $d_{Y}<0,(1-r)^{d_{Y}}-(g-r)^{d_{Y}}<0$ and is minimized at $g=p$. In the case $p<0$ the same considerations apply, but the extremum over $[0,1]$ is at $g=0$ in each case. The proof is completed by noting that for any $p \in[r, 1]$, and $d_{Y}$ of either sign,

$$
\left|(1-r)^{d_{Y}}-(\max \{p, 0\}-r)^{d_{Y}}\right| \leq\left|(1-r)^{d_{Y}}-1_{\{r<0\}}(-r)^{d_{Y}}\right|
$$

To prove Lemma 5.1, first suppose $m \geq 0$. Break the sum $n v_{n m}^{a}$ into components $\sum_{i=0}^{m-1} a_{\text {nim }}^{2}$ and $\sum_{i=-\infty}^{-1} a_{\text {nim }}^{2}$ where the first term is 0 if $m=0$. Note that if $m=[n r]$ for $0 \leq r \leq 1$, then since $i=[n p] \geq 0$, applying Lemma A.2,

$$
\limsup _{n} \frac{1}{n} \sum_{i=0}^{m-1} a_{n i m}^{2} \leq \int_{0}^{r} \bar{A}_{X Y}(r, p)^{2} d p
$$




$$
\begin{aligned}
& = \begin{cases}\frac{(1-r)^{2 d_{Y}+2 d_{X}+1}}{\left(2 d_{X}+1\right) \Gamma\left(d_{X}+1\right)^{2} \Gamma\left(d_{Y}+1\right)^{2}}, & d_{X} \geq 0 \\
\frac{(1-r)^{2 d_{Y}} r^{2 d_{X}+1}}{\left(2 d_{X}+1\right) \Gamma\left(d_{X}+1\right)^{2} \Gamma\left(d_{Y}+1\right)^{2}}, & d_{X}<0\end{cases} \\
& <\infty
\end{aligned}
$$

whereas

$$
\begin{aligned}
\frac{1}{n} \sum_{i=-\infty}^{0} a_{n i m}^{2} & \leq \int_{-\infty}^{0} \bar{A}_{X Y}(r, p)^{2} d p \\
& =\frac{(1-r)^{2 d_{Y}}}{\Gamma\left(1+d_{X}\right)^{2} \Gamma\left(1+d_{Y}\right)^{2}} \int_{0}^{\infty}\left[(1+p)^{d_{X}}-p^{d_{X}}\right]^{2} d p \\
& <\infty
\end{aligned}
$$

noting that $\int_{0}^{\infty}\left[(1+p)^{d}-p^{d}\right]^{2} d p<\infty$ for $|d|<\frac{1}{2}$. (See for example DH Lemma 5.1.) Finally, if $m<0$ and hence $r<0$, by Lemma A.2 there exists $n$ large enough that

$$
\begin{aligned}
\frac{1}{n} \sum_{i=-\infty}^{m} a_{n i m}^{2} & \leq \int_{-\infty}^{r} \bar{A}_{X Y}(r, p)^{2} d p \\
& \leq \frac{\left((1-r)^{d_{Y}}-(-r)^{d_{Y}}\right)^{2}}{\Gamma\left(1+d_{X}\right)^{2} \Gamma\left(1+d_{Y}\right)^{2}} \int_{-r}^{\infty}\left[(1+p)^{d_{X}}-p^{d_{X}}\right]^{2} d p \\
& =O\left((-r)^{2 d_{Y}+2 d_{X}-3}\right) .
\end{aligned}
$$

The argument for Lemma 5.4 is very similar. Letting $i=[n p]$, applying Lemma A.3 leads to

$$
\begin{aligned}
\limsup _{n} \frac{1}{n} \sum_{m=0}^{i-1} e_{n m i}^{2} & \leq \int_{0}^{p} \bar{E}_{X Y}(p, r)^{2} d r \\
& =\frac{\left((1-p)^{d_{X}}-1_{\{p<0\}}(-p)^{d_{X}}\right)^{2}(1-p)^{2 d_{Y}+1}}{\left(2 d_{Y}+1\right) \Gamma\left(d_{X}+1\right)^{2} \Gamma\left(d_{Y}+1\right)^{2}} \\
& <\infty
\end{aligned}
$$

whereas

$$
\frac{1}{n} \sum_{m=-\infty}^{0} e_{n m i}^{2} \leq \int_{-\infty}^{0} \bar{E}_{X Y}(p, r)^{2} d r<\infty
$$

and for $i<0$,

$$
\frac{1}{n} \sum_{m=-\infty}^{i} e_{n m i}^{2} \leq \int_{-\infty}^{p} \bar{E}_{X Y}(p, r)^{2} d r=O\left((-p)^{2 d_{Y}+2 d_{X}-3}\right)
$$

follow exactly as for the proof of Lemma A.2.

\section{A.11 Proof of Lemmas 5.2 and 5.5}

In principle there are three cases to consider, depending on the respective signs of $r$ and $r+\delta$. However, if $r<0$ and $r+\delta>0$ then the interval may be split into subintervals of widths $-r<\delta$ and $\delta+r$ respectively, and treated separately. Showing the cases $r \geq 0$ and $r<r+\delta \leq 0$ is therefore sufficient. The bounding arguments here are essentially the same as those in the proofs of Lemmas 5.1 and 5.4. 
First, for Lemma 5.2,

$$
\begin{aligned}
\limsup _{n} & \frac{1}{n} \sum_{i=[n r]+1}^{[n(r+\delta)]} a_{n i[n(r+\delta)]}^{2} \\
& \leq \int_{r}^{r+\delta} \bar{A}_{X Y}(r+\delta, p)^{2} d p \\
& =\frac{\left[(1-r-\delta)^{d_{Y}}-1_{\{r+\delta<0\}}(-r-\delta)^{d_{Y}}\right]^{2}}{\Gamma\left(1+d_{X}\right)^{2} \Gamma\left(1+d_{Y}\right)^{2}} \int_{r}^{r+\delta}\left[(g-p)^{d_{X}}-1_{\{p<0\}}(-p)^{d_{X}}\right]^{2} d p
\end{aligned}
$$

where $\bar{A}_{X Y}(r, p)$, and $g$, are defined in Lemma A.2.

Case: $r \geq 0$. If $d_{X}>0$, then $g=1$ and

$$
\int_{r}^{r+\delta}(1-p)^{2 d_{X}} d p=\frac{(1-r)^{2 d_{X}+1}-(1-r-\delta)^{2 d_{X}+1}}{2 d_{X}+1}=O(\delta)
$$

otherwise $g=r+\delta$ and

$$
\int_{r}^{r+\delta}(r+\delta-p)^{2 d_{X}} d p=\frac{\delta^{2 d_{X}+1}}{2 d_{X}+1} .
$$

Case: $r<r+\delta \leq 0$. In this case $g=1$ and

$$
\begin{aligned}
\int_{r}^{r+\delta}\left[(1-p)^{d_{X}}-(-p)^{d_{X}}\right]^{2} d p & \leq \int_{r}^{r+\delta}(-p)^{2 d_{X}} d p+\int_{r}^{r+\delta}(1-p)^{2 d_{X}} d p \\
& =O(\delta)
\end{aligned}
$$

since $d_{X} \geq-\frac{1}{2}$. These bounds are independent of $r$, and hold uniformly with respect to $r \in$ $(-\infty, 1]$.

The proof of Lemma 5.5 is very similar, noting that

$$
\begin{aligned}
\limsup \frac{1}{n} \sum_{m=[n p]+1}^{[n(p+\delta)]} e_{n m[n(p+\delta)]}^{2} & \leq \int_{p}^{p+\delta} \bar{E}_{X Y}(p+\delta, r)^{2} d p \\
& =\frac{\left[(1-p-\delta)^{d_{X}}-1_{\{p+\delta>0\}}(-p-\delta)^{d_{X}}\right]^{2}}{\Gamma\left(d_{X}+1\right)^{2} \Gamma\left(d_{Y}+1\right)^{2}} \times \\
& \int_{p}^{p+\delta}\left[(1-r)^{d_{Y}}-1_{\{r<0\}}(-r)^{d_{Y}}\right]^{2} d r \\
& =O(\delta) .
\end{aligned}
$$

\section{A.12 Proof of Lemmas 5.3 and 5.6}

First, for case Lemma 5.3, note that

$$
\begin{array}{r}
a_{n[n p][n(r+\delta)]}-a_{n[n p][n r]}{ }^{n} \frac{n}{K(n)} \sum_{t=\max \{1-[n(r+\delta)], 0\}}^{n-1-[n(r+\delta)]} c_{t}\left(\sum_{s=\max \{1-[n p], 0\}}^{t+[n(r+\delta)]-[n p]} b_{s}\right) \\
-\frac{n}{K(n)} \sum_{t=\max \{1-[n r], 0\}}^{n-1-[n r]} c_{t}\left(\sum_{s=\max \{-[n p], 0\}}^{t+[n r]-[n p]} b_{s}\right)
\end{array}
$$




$$
\begin{gathered}
=-\frac{n}{K(n)} \sum_{t=n-[n(r+\delta)]}^{n-1-[n r]} c_{t}\left(\sum_{s=\max \{-[n p], 0\}}^{t+[n r]-[n p]} b_{s}\right) \\
+\frac{n}{K(n)} \sum_{t=\max \{1-[n r], 0\}}^{n-1-[n(r+\delta)]} c_{t}\left(\sum_{s=t+[n r]-[n p]}^{t+[n(r+\delta)]-[n p]} b_{s}\right) \\
+\frac{n}{K(n)} \sum_{t=\max \{1-[n(r+\delta)], 0\}}^{\max \{1-[n r], 0\}-1} c_{t}\left(\sum_{s=\max \{-[n p], 0\}}^{t+[n(r+\delta)]-[n p]} b_{s}\right) \\
=D_{1 n}^{a}(r, \delta, p)+D_{2 n}^{a}(r, \delta, p)+D_{3 n}^{a}(r, \delta, p)
\end{gathered}
$$

(defining $D_{1 n}^{a}, D_{2 n}^{a}, D_{3 n}^{a}$ ) where an empty sum takes the value 0 by convention, so that $D_{3 n}^{a}=0$ for $r \geq 0$. Now define

$$
\begin{aligned}
& \bar{D}_{1}^{a}(r, \delta, p)=\frac{\left|(1-r)^{d_{Y}}-(1-r-\delta)^{d_{Y}}\right|\left|(1-p)^{d_{X}}-1_{\{p<0\}}(-p)^{d_{X}}\right|}{\Gamma\left(1+d_{X}\right) \Gamma\left(1+d_{Y}\right)} \\
& \bar{D}_{2}^{a}(r, \delta, p)=\frac{\left|(1-r-\delta)^{d_{Y}}-1_{\{r<0\}}(-r)^{d_{Y}}\right|\left|(r+\delta-p)^{d_{X}}-(r-p)^{d_{X}}\right|}{\Gamma\left(1+d_{X}\right) \Gamma\left(1+d_{Y}\right)} \\
& \bar{D}_{3}^{a}(r, \delta, p)=1_{\{r<0\}} \frac{\left|(-r)^{d_{Y}}-(-r-\delta)^{d_{Y}}\right|\left|(\delta-p)^{d_{X}}-(-p)^{d_{X}}\right|}{\Gamma\left(1+d_{X}\right) \Gamma\left(1+d_{Y}\right)} .
\end{aligned}
$$

If $d_{X}>0, d_{Y}>0$ then, using Assumption 2 and Lemma A.1,

$$
\begin{aligned}
& \left|D_{1 n}^{a}(r, \delta, p)\right| \\
& =\frac{1}{n^{2} \Gamma\left(d_{X}\right) \Gamma\left(d_{Y}\right)}\left|\sum_{t=n-[n(r+\delta)]+1}^{n-[n r]}\left(\frac{t}{n}\right)^{d_{Y}-1} \frac{L_{Y}(t)}{L_{Y}(n)}\left(\sum_{s=\max \{-[n p], 0\}+1}^{t+[n r]-[n p]+1}\left(\frac{s}{n}\right)^{d_{X}-1} \frac{L_{X}(t)}{L_{X}(n)}\right)\right| \\
& \leq \frac{1}{\Gamma\left(d_{X}\right) \Gamma\left(d_{Y}\right)}\left|\frac{1}{n} \sum_{t=n-[n(r+\delta)]+1}^{n-[n r]}\left(\frac{t}{n}\right)^{d_{Y}-1}\right|\left|\frac{1}{n} \sum_{s=\max \{-[n p], 0\}+1}^{n-[n p]+1}\left(\frac{s}{n}\right)^{d_{X}-1}\right| \\
& =\bar{D}_{1}^{a}(r, \delta, p)+O\left(n^{\max \left\{-d_{X},-d_{Y}\right\}}\right) ; \\
& \left|D_{2 n}^{a}(r, \delta, p)\right| \\
& =\frac{1}{n^{2} \Gamma\left(d_{X}\right) \Gamma\left(d_{Y}\right)}\left|\sum_{t=\max \{1-[n r], 0\}+1}^{n-[n(r+\delta)]}\left(\frac{t}{n}\right)^{d_{Y}-1} \frac{L_{Y}(t)}{L_{Y}(n)}\left(\sum_{s=t+[n r]-[n p]+1}^{t+[n(r+\delta)]-[n p]+1}\left(\frac{s}{n}\right)^{d_{X}-1} \frac{L_{X}(t)}{L_{X}(n)}\right)\right| \\
& \leq \frac{1}{\Gamma\left(d_{X}\right) \Gamma\left(d_{Y}\right)}\left|\frac{1}{n} \sum_{t=\max \{1-[n r], 0\}+1}^{n-[n(r+\delta)]}\left(\frac{t}{n}\right)^{d_{Y}-1}\right|\left|\frac{1}{n} \sum_{s=[n r]-[n p]}^{[n(r+\delta)]-[n p]}\left(\frac{s}{n}\right)^{d_{X}-1}\right| \\
& =\bar{D}_{2}^{a}(r, \delta, p)+O\left(n^{-d_{X}}\right) \text {; } \\
& \left|D_{3 n}^{a}(r, \delta, p)\right| \\
& =\frac{1_{\{r<0\}}}{n^{2} \Gamma\left(d_{X}\right) \Gamma\left(d_{Y}\right)}\left|\sum_{t=-\max \{1-[n(r+\delta)], 0\}+1}^{1-[n r]}\left(\frac{t}{n}\right)^{d_{Y}-1} \frac{L_{Y}(t)}{L_{Y}(n)}\left(\sum_{s=1-[n p]}^{t+[n(r+\delta)]-[n p]+1}\left(\frac{s}{n}\right)^{d_{X}-1} \frac{L_{X}(t)}{L_{X}(n)}\right)\right| \\
& \leq \frac{1_{\{r<0\}}}{\Gamma\left(d_{X}\right) \Gamma\left(d_{Y}\right)}\left|\frac{1}{n} \sum_{t=1-[n(r+\delta)]}^{1-[n r]}\left(\frac{t}{n}\right)^{d_{Y}-1}\right|\left|\frac{1}{n} \sum_{s=1-[n p]}^{2+[n \delta]-[n p]}\left(\frac{s}{n}\right)^{d_{X}-1}\right|
\end{aligned}
$$




$$
=\bar{D}_{3}^{a}(r, \delta, p)+O\left(n^{-d_{Y}}\right) .
$$

The cases having $d_{Y} \leq 0$ and/or $d_{X} \leq 0$ require modification of these formulae on the lines of equation (A-13). Terms of the form $\left(\frac{t}{n}\right)^{d_{Y}-1}$ and $\left(\frac{s}{n}\right)^{d_{X}-1}$ are replaced respectively with terms of the form $\left(\frac{t+1}{n}\right)^{d_{Y}}-\left(\frac{t}{n}\right)^{d_{Y}}$ and $\left(\frac{s+1}{n}\right)^{d_{X}}-\left(\frac{s}{n}\right)^{d_{X}}$. The approximation error rates are modified in the same manner, with $-1-d_{Y}$ replacing $-d_{Y}$ when $d_{Y}<0$ and $-1-d_{X}$ replacing $-d_{X}$ when $d_{X}<0$. However, note that although the signs of the sums depend on the signs of $d_{Y}$ and $d_{X}$, the indicated bounds hold in all cases. We therefore have that there exists $n$ large enough that

$$
\left|a_{n[n p][n(r+\delta)]}-a_{n[n p][n r]}\right| \leq \bar{D}_{1}^{a}(r, \delta, p)+\bar{D}_{2}^{a}(r, \delta, p)+\bar{D}_{3}^{a}(r, \delta, p) .
$$

Therefore,

$$
\begin{aligned}
\limsup _{n} \frac{1}{n} \sum_{i=-\infty}^{[n r]} & \left(a_{n i[n(r+\delta)]}-a_{n i[n r]}\right)^{2} \\
& \left.\left.\leq \int_{-\infty}^{r} \bar{D}_{1}^{a}(r, \delta, p)^{2} d p+\int_{-\infty}^{r} \bar{D}_{2}^{a}(r, \delta, p)\right)^{2} d p+\int_{-\infty}^{r} \bar{D}_{3}^{a}(r, \delta, p)\right)^{2} d p
\end{aligned}
$$

where

$$
\begin{aligned}
\int_{-\infty}^{r} \bar{D}_{1}^{a}(r, \delta, p)^{2} d p & \leq \frac{\left.(1-r)^{d_{Y}}-(1-r-\delta)^{d_{Y}}\right)^{2}}{\Gamma\left(1+d_{X}\right)^{2} \Gamma\left(1+d_{Y}\right)^{2}} \int_{-\infty}^{r}\left[(1-p)^{d_{X}}-1_{\{p<0\}}(-p)^{d_{X}}\right]^{2} d p \\
& =O\left(\delta^{2}\right) \\
\left.\int_{-\infty}^{r} \bar{D}_{2}^{a}(r, \delta, p)\right)^{2} d p & \leq \frac{\left[(1-r-\delta)^{d_{Y}}-1\{r<0\}(-r)^{d_{Y}}\right]^{2}}{\Gamma\left(1+d_{X}\right)^{2} \Gamma\left(1+d_{Y}\right)^{2}} \int_{-\infty}^{r}\left[(r+\delta-p)^{d_{X}}-(r-p)^{d_{X}}\right]^{2} d p \\
= & O\left(\delta^{2 d_{X}+1}\right)
\end{aligned}
$$

and if $r<0$,

$$
\begin{aligned}
\left.\int_{-\infty}^{r} \bar{D}_{3}^{a}(r, \delta, p)\right)^{2} d p & \leq \frac{\left[(-r)^{d_{Y}}-(-r-\delta)^{d_{Y}}\right]^{2}}{\Gamma\left(1+d_{X}\right)^{2} \Gamma\left(1+d_{Y}\right)^{2}} \int_{-\infty}^{r}\left[(\delta-p)^{d_{X}}-(-p)^{d_{X}}\right]^{2} d p \\
& =O\left(\delta^{2 d_{X}+3}\right) .
\end{aligned}
$$

These bounds are independent of $r$, and hold uniformly with respect to $r \in(-\infty, 1]$.

For Lemma 5.6, we have from (4.4)

$$
\begin{aligned}
e_{n[n r][n(p+\delta)]} & -e_{n[n r][n p]} \\
= & \frac{n}{K(n)} \sum_{s=\max \{1-[n(p+\delta)], 0\}}^{n-1-[n(p+\delta)]} b_{s}\left(\sum_{t=s+[n(p+\delta)]+1-[n r]}^{n-[n r]} c_{t}\right) \\
& -\frac{n}{K(n)} \sum_{s=\max \{0,1-[n p]\}}^{n-1-[n p]} b_{s}\left(\sum_{t=s+[n p]+1-[n r]}^{n-[n r]} c_{t}\right) \\
=-\frac{n}{K(n)} & \sum_{s=n-[n(p+\delta)]}^{n-1-[n p]} b_{s}\left(\sum_{t=s+[n(p+\delta)]+1-[n r]}^{n-[n r]} c_{t}\right)
\end{aligned}
$$




$$
\begin{gathered}
\frac{n}{K(n)} \sum_{s=\max \{0,1-[n p]\}}^{n-1-[n(p+\delta)]} b_{s}\left(\sum_{t=s+[n p]+1-[n r]}^{s+[n(p+\delta)]+1-[n r]} c_{t}\right) \\
+1_{\{p<0\}} \frac{n}{K(n)} \sum_{s=\max \{0,1-[n(p+\delta)]\}} b_{s}\left(\sum_{t=s+[n(p+\delta)]+1-[n r]}^{n-[n r]} c_{t}\right) \\
=D_{1 n}^{e}(p, \delta, r)+D_{2 n}^{e}(p, \delta, r)+D_{3 n}^{e}(p, \delta, r) .
\end{gathered}
$$

Similarly to the previous case, there exists $n$ large enough that

$$
\begin{aligned}
\left|D_{1 n}^{e}(p, \delta, r)\right| & \leq \bar{D}_{1}^{e}(p, \delta, r) \\
& =\frac{\left|(1-p)^{d_{X}}-(1-p-\delta)^{d_{X}}\right|\left|(1-r)^{d_{Y}}-(1+\delta-r)^{d_{Y}}\right|}{\Gamma\left(1+d_{X}\right) \Gamma\left(1+d_{Y}\right)}
\end{aligned}
$$

$$
\begin{aligned}
\left|D_{2 n}^{e}(p, \delta, r)\right| & \leq \bar{D}_{2}^{e}(p, \delta, r) \\
& =\frac{\left|(1-p-\delta)^{d_{X}}-1_{\{p<0\}}(-p)^{d_{X}}\right|\left|(\max \{p, 0\}-\delta-r)^{d_{Y}}-(\max \{p, 0\}-r)^{d_{Y}}\right|}{\Gamma\left(1+d_{X}\right) \Gamma\left(1+d_{Y}\right)}
\end{aligned}
$$

and

$$
\begin{aligned}
\left|D_{3 n}^{e}(p, \delta, r)\right| & \leq \bar{D}_{2}^{e}(p, \delta, r) \\
& =1_{\{p<0\}} \frac{\left|(-p)^{d_{X}}-(-p-\delta)^{d_{X}}\right|\left|(1-r)^{d_{Y}}-(-r)^{d_{Y}}\right|}{\Gamma\left(1+d_{X}\right) \Gamma\left(1+d_{Y}\right)}
\end{aligned}
$$

Therefore, similarly to the previous case,

$$
\begin{aligned}
\limsup _{n} \frac{1}{n} \sum_{m=-\infty}^{[n p]}\left(e_{n m[n(p+\delta)]}-e_{n m[n p]}\right)^{2} & \leq \int_{-\infty}^{p} \bar{D}_{1}^{e}(p, \delta, r)^{2} d p+\int_{-\infty}^{p} \bar{D}_{2}^{e}(p, \delta, r)^{2} d p \int_{-\infty}^{p} \bar{D}_{3}^{e}(p, \delta, r)^{2} d p \\
& =O\left(\delta^{2 d_{Y}+1}\right)
\end{aligned}
$$

where the bound is independent of $p$, and holds uniformly in $p \in(-\infty, 1]$.

\section{A.13 Proof of Theorems 5.2 and 5.5}

We argue for the exemplar case of $Q_{n}^{N}$. The proof for $H_{n}^{N}$ is essentially identical, after swapping $H$ for $Q, w$ for $u$ and $e$ for $a$, and also substituting the lemmas from Section 5.2 for those of Section 5.1

The proof follows the lines of Theorem 3.1 of Davidson and de Jong (2000) (henceforth, DDJ), which in turn is based on Theorem 3.1 of DJD. Similarly to the DDJ theorem, the sample period is changed from $1, \ldots, K_{n}$ to $-N n, \ldots, n$. For the finite dimensional distributions, we apply the CLT of de Jong (1997). Since the $u_{i}$ are assumed independent, this is simply a matter of establishing a counterpart of the Lindeberg condition for the process $q_{n m}^{N}$.

To translate the conditions of the present setup into those of the DDJ model, note that the process in question has increments

$$
Q_{n}^{N}(r+\delta)-Q_{n}^{N}(r)=\frac{1}{\sqrt{n}} \sum_{i=[n r]+1}^{[n(r+\delta)]} a_{n i[n(r+\delta)]} u_{i}+\frac{1}{\sqrt{n}} \sum_{i=-N n}^{[n r]}\left(a_{n i[n(r+\delta)]}-a_{n i[n r]}\right) u_{i} .
$$


The notations of this paper and DDJ may be connected by matching $t, \xi$ and $\xi^{\prime}$ in DDJ with $i, r+\delta$ and $r$ in this paper, respectively, and so noting that the quantities denoted $a_{n t}\left(\xi, \xi^{\prime}\right)$ in DDJ, equation (3.1), correspond in the present notation to $a_{n i[n(r+\delta)]}$ for $i>[n r]$, and to $a_{n i[n(r+\delta)]}-a_{n i[n r]}$ for $-\infty<i \leq[n r]$ otherwise. Since the shocks $u_{i}$ are i.i.d by Assumption 1, bounds on the variances of the increments in (A-26) are found from Lemmas 5.1, 5.2 and 5.3. The finite dimensional distributions of the variates $Q_{n}^{N}(r)$ can be determined from Theorem 3.1 of DJD. Note that the variates denoted $X_{n t}$ in that theorem correspond in the present case to either $n^{-1 / 2} a_{n i[n(r+\delta)]} u_{i}$ or to $n^{-1 / 2}\left(a_{n i[n(r+\delta)]}-a_{n i[n r]}\right) u_{i}$ for $-n N \leq i \leq[n(r+\delta)]$ and, given the conditions specified in our Assumptions 1 and 2, are sufficient for DJD's Assumption 1. Note further that $r$ is fixed in each application of this theorem, that condition (3.2) in DJD holds in the present case by Lemma 5.1, and that condition 3.3 in DJD holds in the present case by Lemmas 5.2 and 5.3. Finally, to show the tightness of the sequence of measures the argument in DDJ, Theorem 3.1, can be applied with appropriate substitutions. (See also the addendum to this theorem in Davidson (2001)). Noting the equivalences set out above, the condition corresponding to DDJ (B-35) follows directly from Lemmas 5.2 and 5.3.

\section{A.14 Proof of Theorems 5.4 and 5.7}

First note that

$$
\begin{aligned}
\frac{1}{n^{2}} \mathrm{E}\left(\sum_{m=-N n}^{n-1}\left(q_{n m}-q_{n m}^{N}\right) w_{m+1}\right)^{2} & =\frac{1}{n^{2}} \mathrm{E}\left(\sum_{m=-N n}^{n-1} \sum_{i=-\infty}^{-N n} u_{i} a_{n i m} w_{m+1}\right)^{2} \\
& =\omega_{u u} \omega_{w w} \frac{1}{n^{2}} \sum_{m=-N n}^{n-1} \sum_{i=-\infty}^{-N n} a_{n i m}^{2}
\end{aligned}
$$

Copying the argument from the proof of Lemma 5.1, note that

$$
\frac{1}{n^{2}} \sum_{m=-N n}^{n-1} \sum_{i=-\infty}^{-N n} a_{n i m}^{2}=\int_{-N}^{1}\left(\int_{-\infty}^{-N} A_{X Y}(r, p)^{2} d p\right) d r+o(1)
$$

as $n \rightarrow \infty$ where

$$
\begin{aligned}
\int_{-N}^{1}\left(\int_{-\infty}^{-N} A_{X Y}(r, p)^{2} d p\right) d r & \leq \int_{-N}^{1}\left(\int_{-\infty}^{-N} \bar{A}_{X Y}(r, p)^{2} d p\right) d r \\
& =\frac{1}{\Gamma\left(1+d_{X}\right)^{2} \Gamma\left(d_{Y}+1\right)^{2}} \int_{-N}^{1}\left((1-r)^{d_{Y}}-(-r)^{d_{Y}}\right)^{2} d r \\
& =O\left(N^{2 d_{Y}+2 d_{X}-2}\right) .
\end{aligned}
$$

Next,

$$
\begin{aligned}
\frac{1}{n^{2}} \mathrm{E}\left(\sum_{m=-\infty}^{-N n-1} q_{n m} w_{m+1}\right)^{2} & =\omega_{u u} \omega_{w w} \frac{1}{n^{2}} \sum_{m=-\infty}^{-N n-1} \sum_{i=-\infty}^{m} a_{n i m}^{2} \\
& =\int_{-\infty}^{-N} \int_{-\infty}^{r} A_{X Y}(r, p)^{2} d p d r+o(1)
\end{aligned}
$$


as $n \rightarrow \infty$, and by previous arguments, letting $K$ denote a finite positive constant, we have

$$
\begin{aligned}
& \int_{-\infty}^{N}\left(\int_{-\infty}^{-r} A_{X Y}(r, p)^{2} d p\right) d r \leq \int_{-\infty}^{N}\left(\int_{-\infty}^{-r} A_{X Y}(r, p)^{2} d p\right) d r \\
& =\frac{1}{\Gamma\left(1+d_{X}\right)^{2} \Gamma\left(d_{Y}+1\right)^{2}} \int_{-\infty}^{-N}\left((1-r)^{d_{Y}}-(-r)^{d_{Y}}\right)^{2} \\
& \quad \times \int_{-r}^{\infty}\left[(1+p)^{d_{X}}-p^{d_{X}}\right]^{2} d p d r \\
& \leq \frac{K}{\Gamma\left(1+d_{X}\right)^{2} \Gamma\left(d_{Y}+1\right)^{2}} \int_{-\infty}^{-N}\left((1-r)^{d_{Y}}-(-r)^{d_{Y}}\right)^{2}(-r)^{2 d_{X}-2} d r \\
& =O\left(N^{\left.2 d_{Y}+2 d_{X}-3\right) .}\right.
\end{aligned}
$$

The case of Theorem 5.7 is essentially similar.

\section{References}

Abramowitz, M and I A. Stegun (1972) Handbook of Mathematical Functions 10th Edn. Dover: New York.

Billingsley, P. (1968) Convergence of Probability Measures. New York: John Wiley \& Sons.

Chan, N. H. and N. Terrin (1995) Inference for unstable long-memory processes with applications to fractional unit root autoregressions. Annals of Statistics 25(5) 1662-1683.

Davidson, J. (1994) Stochastic Limit Theory. Oxford University Press.

Davidson, J. and R. M. de Jong (2000) The functional central limit theorem and convergence to stochastic integrals II: fractionally integrated processes. Econometric Theory 16(5) 643-666.

Davidson, J. (2001) Addendum to "The functional central limit theorem and convergence to stochastic integrals II: fractionally integrated processes" at http://www.people.ex.ac.uk/ jehd201/fcltfiadd.pdf

Davidson, J. (2006) Alternative bootstrap procedures for testing cointegration in fractionally integrated processes Journal of Econometrics 133 (2) 741-777.

Davidson, J. and N. Hashimzade (2008) Alternative frequency and time domain versions of fractional Brownian motion. Econometric Theory 24(1), 256-293.

De Jong, R. M. and J. Davidson (2000) The functional central limit theorem and convergence to stochastic integrals I: the weakly dependent case. Econometric Theory 16(5) 621-642

Johansen, S. (1991) Estimation and hypothesis testing of cointegration in Gaussian vector autoregressive models. Econometrica 59, 1551-80.

Kurtz, T.G. and and P. E. Protter (1991) Weak Limit Theorems for Stochastic Integrals and Stochastic Differential Equations. Annals of Probability 19(3) 1035-1070.

Kurtz, T.G. and and P. E. Protter (1995) Weak convergence of stochastic integrals and differential equations, in Probabilistic Models for Nonlinear Partial Differential Equations: Lectures Given at the 1st Session of the Centro Internazionale Matematico Estivo (C.I.M.E.), by K. Graham, T.G. Kurtz, S. Meleard, P.E. Protter, M. Pulverenti, and D. Talay. Lecture Notes in Mathematics 1627, Springer Verlag.

Lettau, M. and S. Ludvigson (2001) Consumption, aggregate wealth and expected stock returns. Journal of Finance LVI(3), 815-849. 
Mandelbrot, B. B. and J. W. van Ness (1968) Fractional Brownian motions, fractional noises and applications. SIAM Review 10(4) 422-437.

Phillips, P. C. B. (1987) Time series regression with a unit root Econometrica 55, 277-301.

Phillips, P. C. B., and B. E. Hansen (1990) Statistical inference in instrumental variables regression with I(1) processes, Review of Economic Studies 57, 99-125.

Saikkonen, P. (1991) Asymptotically efficient estimation of cointegration regressions, Econometric Theory 7, 1-21.

Sowell, F. (1990) The fractional unit root distribution, Econometrica 58, 495-505. 